\title{
Lattice Abelian-Higgs model with noncompact gauge fields
}

\author{
Claudio Bonati $\odot,{ }^{1}$ Andrea Pelissetto $\odot,{ }^{2}$ and Ettore Vicari $\odot^{1}$ \\ ${ }^{1}$ Dipartimento di Fisica dell'Università di Pisa and INFN, Largo Pontecorvo 3, I-56127 Pisa, Italy \\ ${ }^{2}$ Dipartimento di Fisica dell'Università di Roma Sapienza and INFN Sezione di Roma I, I-00185 Roma, Italy
}

(Received 21 October 2020; revised 15 December 2020; accepted 21 January 2021; published 2 February 2021)

\begin{abstract}
We consider a noncompact lattice formulation of the three-dimensional electrodynamics with $N$-component complex scalar fields, i.e., the lattice Abelian-Higgs model with noncompact gauge fields. For any $N \geqslant 2$, the phase diagram shows three phases differing for the behavior of the scalar-field and gauge-field correlations: The Coulomb phase (short-ranged scalar and long-ranged gauge correlations), the Higgs phase (condensed scalarfield and gapped gauge correlations), and the molecular phase (condensed scalar-field and long-ranged gauge correlations). They are separated by three transition lines meeting at a multicritical point. Their nature depends on the phases they separate, and on the number $N$ of components of the scalar field. In particular, the Coulombto-molecular transition line (where gauge correlations are irrelevant) is associated with the Landau-GinzburgWilson $\Phi^{4}$ theory sharing the same SU(N) global symmetry but without explicit gauge fields. On the other hand, the Coulomb-to-Higgs transition line (where gauge correlations are relevant) turns out to be described by the continuum Abelian-Higgs field theory with explicit gauge fields. Our numerical study is based on finite-size scaling analyses of Monte Carlo simulations with $C^{*}$ boundary conditions (appropriate for lattice systems with noncompact gauge variables, unlike periodic boundary conditions), for several values of $N$, i.e., $N=2,4,10,15$, and 25 . The numerical results agree with the renormalization-group predictions of the continuum field theories. In particular, the Coulomb-to-Higgs transitions are continuous for $N \gtrsim 10$, in agreement with the predictions of the Abelian-Higgs field theory.
\end{abstract}

DOI: 10.1103/PhysRevB.103.085104

\section{INTRODUCTION}

Models of scalar fields with $\mathrm{U}(1)$ gauge symmetry and $\mathrm{SU}(N)$ global symmetry emerge as effective theories of superconductors, superfluids, and of quantum $\mathrm{SU}(N)$ antiferromagnets [1-8]. In particular, three-dimensional (3D) classical $\mathrm{U}(1)$ gauge models with $N=2$ supposedly describe the transition between the Néel and the valence-bond-solid state in two-dimensional antiferromagnetic SU(2) quantum systems [9-16] that represent the paradigmatic models for the so-called deconfined quantum criticality [17].

This class of quantum models and their classical counterparts have been extensively studied with the purpose of identifying the nature of their different phases and transitions. It has been realized that topological aspects, like the Berry phase, monopoles, or the compact or noncompact nature of the gauge fields, play a crucial role in determining the nature of the transition. For example, the critical behavior of the simplest classical model with U(1) gauge symmetry, the lattice $\mathrm{CP}^{N-1}$ model, drastically depends on the presence or absence of topological defects [18-20], such as monopoles, both for large and small values of $N$, in particular for $N=2$. Analogous differences emerge in the behavior of compact and noncompact lattice formulations of scalar electrodynamics, i.e., of the multicomponent Abelian-Higgs (AH) model. In particular, for $N=2$, theoretical and numerical investigations of classical and quantum transitions, which are expected to be in the same universality class as those occurring in non- compact scalar electrodynamics, have provided evidence of weakly first-order or continuous transitions belonging to a new universality class (see, e.g., Refs. [7,9-18,21-36]).

Here we present a numerical study of the phase diagram of the lattice AH model with noncompact gauge fields, for several values of $N$, the number of components of the complex scalar field. The noncompact model (in which there are no monopoles) is indeed the one of interest in many condensed-matter physics applications, since the presence of Berry phases in the quantum setting requires to suppress monopoles (which are directly related to the Berry phases in the quantum case [37]) (see, e.g., Ref. [6] and references therein). Our study confirms the existence of important differences with the compact AH model [38], for both small and large values of $N$.

In Fig. 1 we sketch the phase diagram of the noncompact lattice AH model with unit-length $N$-component scalar fields. For any $N \geqslant 2$ the phase diagram is characterized by three phases. There is a Coulomb phase, in which the global SU $(N)$ symmetry is unbroken and the electromagnetic correlations are long ranged. The other two phases are characterized by the breaking of the $\mathrm{SU}(N)$ symmetry. They are distinguished by the behavior of the gauge modes. In the Higgs phase, electromagnetic correlations are gapped, while in the molecular phase the electromagnetic field is ungapped. The Coulomb, molecular, and Higgs phases are separated by three different transition lines meeting at one point of the phase diagram. The nature of the transition lines is different, due to the fact 


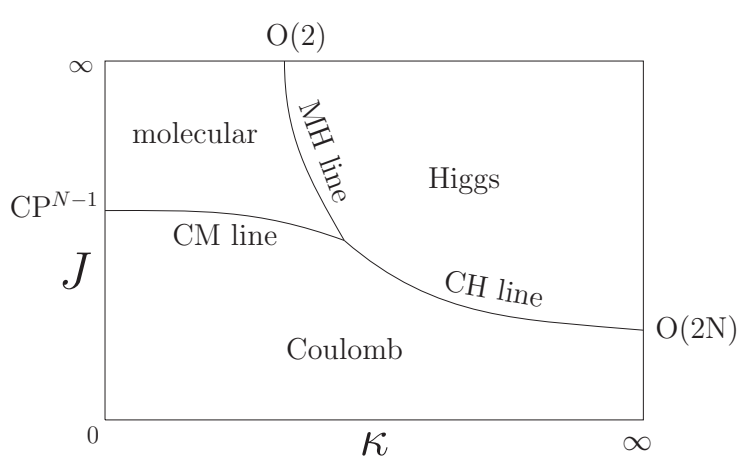

FIG. 1. Sketch of the phase diagram of the lattice AH model with noncompact gauge fields and unit-length $N$-component complex scalar fields, for generic $N \geqslant 2$. Three transition lines can be identified: The Coulomb-to-Higgs $(\mathrm{CH})$ line between the Coulomb and Higgs phases, the Coulomb-to-molecular (CM) line, and the molecular-to-Higgs $(\mathrm{MH})$ line. They are continuous or of first order depending on the value of $N$ (see Sec. IV for details). We also report the models emerging in some limiting cases: The $\mathrm{CP}^{N-1}$ model for $\kappa=0$, the $\mathrm{O}(2 N)$ vector model for $\kappa \rightarrow \infty$, and the inverted XY or $\mathrm{O}(2)$ model for $J \rightarrow \infty$.

that they separate different phases. Moreover, their nature crucially depends on the number $N$ of components.

Our numerical study allows us to characterize the nature of the different transition lines. For large $N$, the critical behavior along the Coulomb-to-Higgs $(\mathrm{CH})$ transition line belongs to the universality class associated with the stable fixed point (FP) of the multicomponent AH field theory, which predicts a continuous transition only for a large number of components (we present numerical evidence of continuous transitions for $N \geqslant 10$ ), and in particular in the large- $N$ limit. For small $N$, instead, the $\mathrm{CH}$ line is characterized by weak first-order transitions (this is the case for $N=2,4$ that we investigate numerically). Along the Coulomb-to-molecular (CM) transition line, gauge correlations do not play any particular role. Numerical results are consistent with the predictions of the Landau-Ginzburg-Wilson (LGW) $\Phi^{4}$ field theory with $\mathrm{SU}(N)$ global symmetry and without explicit gauge fields. Therefore, for $N=2 \mathrm{CM}$ transitions are continuous and belong to the $\mathrm{O}(3)$ vector universality class, while they are expected to be of first order for any larger value of $N$. Finally, the molecular-toHiggs (MH) transitions are essentially related to the behavior of the gauge correlations. They are expected to belong to the universality class of the inverted XY model, for any $N$.

The paper is organized as follows. In Sec. II we introduce the lattice AH model. In Sec. III we discuss the fieldtheoretical models that may be relevant for the phase transitions of the model. In Sec. IV we present the possible scenarios for the phase diagram and for the nature of the transition lines, focusing on some limits of the Hamiltonian parameters. Section V presents our numerical results, for $N=2,4,10,15$, and 25. Finally, we summarize and draw our conclusions in Sec. VI. In the Appendix we discuss the pathologies of periodic boundary conditions in systems with noncompact gauge variables (averages of gauge-invariant noncompact Polyakov lines are not defined). To overcome this problem, we consider $C^{*}$ boundary conditions $[39,40]$, which allow us to obtain a rigorous definition of the model in a finite volume.

\section{THREE-DIMENSIONAL LATTICE ABELIAN-HIGGS MODELS}

We consider $d$-dimensional systems characterized by a global $\mathrm{SU}(N)$ symmetry and a local $\mathrm{U}(1)$ gauge symmetry. A paradigmatic quantum field theory with these symmetries is the multicomponent scalar electrodynamics, or AH field theory, in which an $N$-component complex scalar field $\boldsymbol{\Phi}$ is minimally coupled to the electromagnetic field $A_{\mu}$. The corresponding continuum Lagrangian reads

$$
\mathcal{L}=\left|D_{\mu} \boldsymbol{\Phi}\right|^{2}+r \boldsymbol{\Phi}^{*} \boldsymbol{\Phi}+\frac{1}{6} u\left(\boldsymbol{\Phi}^{*} \boldsymbol{\Phi}\right)^{2}+\frac{1}{4 g^{2}} F_{\mu \nu}^{2},
$$

where $F_{\mu \nu} \equiv \partial_{\mu} A_{\nu}-\partial_{\nu} A_{\mu}$ and $D_{\mu} \equiv \partial_{\mu}+i A_{\mu}$.

In the following we consider lattice models that are formal discretizations of the continuum AH model. In particular, one may consider lattice models that differ on the topologicalcompact or noncompact-nature of the gauge fields. We consider unit-length $N$-component complex variables $z_{x}$ associated with each site of a cubic lattice and gauge fields associated with the lattice links. The lattice Hamiltonian reads

$$
H=H_{z}+H_{g},
$$

with

$$
H_{z}=-J N \sum_{x, \mu} 2 \operatorname{Re}\left(\bar{z}_{x} \cdot \lambda_{x, \mu} z_{x+\hat{\mu}}\right),
$$

where the sum runs over all links of the cubic lattice, and $\lambda_{x, \mu}$ is a complex gauge field with $\left|\lambda_{x, \mu}\right|=1$. In compact formulations the link phase $\lambda_{x, \mu}$ is the fundamental gauge variable. The corresponding simplest gauge Hamiltonian reads

$$
H_{g}=-\kappa \sum_{\boldsymbol{x}, \mu>\nu} \operatorname{Re}\left(\lambda_{\boldsymbol{x}, \mu} \lambda_{\boldsymbol{x}+\hat{\mu}, \nu} \bar{\lambda}_{\boldsymbol{x}+\hat{v}, \mu} \bar{\lambda}_{\boldsymbol{x}, \nu}\right),
$$

where the sum is over the lattice plaquettes and $\kappa$ plays the role of inverse gauge coupling. The partition function is $Z=$ $\sum_{\{z, \lambda\}} e^{-\beta H}$.

In noncompact formulations the fundamental gauge variable is the real vector field $A_{\boldsymbol{x}, \mu}$ and

$$
\lambda_{x, \mu}=e^{i A_{x, \mu}} .
$$

In this case, the gauge Hamiltonian $H_{g}$ can be straightforwardly derived from the continuum theory (1), by replacing the tensor field $F_{\mu \nu}(\boldsymbol{x})$ with its discretized lattice counterpart, i.e.,

$$
H_{g}=\frac{\kappa}{2} \sum_{\boldsymbol{x}, \mu>v}\left(\Delta_{\hat{\mu}} A_{\boldsymbol{x}, v}-\Delta_{\hat{v}} A_{\boldsymbol{x}, \mu}\right)^{2} .
$$

Here the sum runs over all plaquettes, $\Delta_{\hat{\mu}}$ denotes the discretized derivative along $\hat{\mu}$ (i.e., $\Delta_{\hat{\mu}} A_{x} \equiv A_{x+\hat{\mu}}-A_{x}$ ), and $\kappa \geqslant 0$ corresponds to the inverse gauge coupling $1 / g^{2}$ of the continuum theory (1). The partition function reads

$$
Z=\sum_{\{z, A\}} e^{-\beta H}
$$

In the following we rescale $J$ and $\kappa$ by $\beta$, thus formally setting $\beta=1$. 
It is important to note that, at variance with the compact case, the partition function (7) is only formally defined. Because of gauge invariance, there is an infinite number of zero modes; therefore, $Z=\infty$. As discussed in detail in the Appendix, by an appropriate choice of boundary conditions and by restricting our attention to gauge-invariant observables, we can make $Z$, as well as any gauge-invariant average, well defined. This is, of course, of crucial importance for the numerical computation. Note that periodic boundary conditions cannot be used for the noncompact model. Indeed, in this case the Polyakov loops in terms of the noncompact variables are not bounded and never thermalize; thus, even gauge-invariant observables are ill defined.

In the following we study the phase diagram and the transition lines of the noncompact model (6). Appropriate order parameters can be defined in terms of the $z_{x}$ and $A_{x, \mu}$ fields. In our study we focus on the correlations of the gauge-invariant bilinear operator

$$
Q_{x}^{a b}=\bar{z}_{x}^{a} z_{x}^{b}-\frac{1}{N} \delta^{a b},
$$

which is an Hermitian and traceless $N \times N$ matrix that transforms as $Q_{x} \rightarrow U^{\dagger} Q_{x} U$ under the global $\mathrm{SU}(N)$ transformations.

\section{FIELD-THEORETICAL APPROACHES}

One of the motivations of this work is that of understanding the relation between the phase diagram of 3D lattice Abelian gauge models and the renormalization-group (RG) flow of the continuum AH model (1), which has been studied within the $\varepsilon \equiv 4-d$ expansion framework [41-43], using the functional $\mathrm{RG}$ approach [44], and in the large- $N$ limit $[41,45-48]$. One expects that the 3D RG flow of the continuum AH model describes some critical transitions occurring in 3D statistical systems characterized by an Abelian gauge symmetry and a global $\mathrm{SU}(N)$ symmetry. However, as far as we know, the correspondence between the transition lines observed in lattice systems and the fixed points of the continuum AH model has not been fully clarified yet.

\section{A. RG flow of the AH field theory}

In the $\varepsilon$-expansion framework, the RG flow is determined by the $\beta$ functions associated with the renormalized couplings $u$ and $f \equiv g^{2}$ appearing in the Lagrangian Eq. (1). One-loop computations give [41]

$$
\begin{aligned}
& \beta_{u} \equiv \mu \frac{\partial u}{\partial \mu}=-\varepsilon u+(N+4) u^{2}-18 u f+54 f^{2}, \\
& \beta_{f} \equiv \mu \frac{\partial f}{\partial \mu}=-\varepsilon f+N f^{2},
\end{aligned}
$$

whew we used rescaled couplings $u \rightarrow u /\left(24 \pi^{2}\right)$ and $f \rightarrow$ $f /\left(24 \pi^{2}\right)$ to simplify the equations. A stable fixed point is present only for

$$
N \geqslant N_{4}=90+24 \sqrt{15} \approx 183 .
$$

It is located at

$$
u^{*}=\frac{N+18+\sqrt{N^{2}-180 N-540}}{2 N(N+4)} \varepsilon, \quad f^{*}=\frac{\varepsilon}{N} .
$$

More generally, in generic dimensions $d=4-\varepsilon$, a stable fixed point exists only for $N>N_{c}(\varepsilon)$. This implies that 3D lattice AH models may undergo a continuous transition associated with the AH stable fixed point only if

$$
N>N_{c} \equiv N_{c}(1) .
$$

The critical number of components, $N_{c}(\varepsilon)$, has been determined to four loops [43]:

$$
N_{c}(\varepsilon)=N_{4}\left[1-1.752 \varepsilon+0.789 \varepsilon^{2}+0.362 \varepsilon^{3}+O\left(\varepsilon^{4}\right)\right] .
$$

The large coefficients of expansion (13) make a reliable 3D (i.e., for $\varepsilon=1$ ) estimate quite problematic. Nevertheless, by means of a resummation of the expansion that takes somehow into account two-dimensional results, Ref. [43] obtained $N_{c}=$ 12.2(3.9) in three dimensions, which confirms the absence of a stable fixed point for small values of $N$.

In the limit $\kappa \rightarrow \infty$, the gauge fields order so that $\lambda_{x, \mu}=1$. The lattice AH model becomes equivalent to the symmetric $\mathrm{O}(2 N)$ vector theory. Therefore, for large $\kappa$, one expects significant crossover effects, which increase as $\kappa$ increases, due to the nearby $\mathrm{O}(2 N)$ critical transition. In the continuum $\mathrm{AH}$ model, the crossover is controlled by the RG flow in the vicinity of the $\mathrm{O}(2 N)$ fixed point,

$$
u_{\mathrm{O}(2 N)}^{*}=\frac{1}{N+4} \varepsilon, \quad f=0 .
$$

This fixed point exists for any $N$ and is always unstable. The analysis of the stability matrix $\Omega_{i j}=\partial \beta_{i} / \partial g_{j}$ shows that it has a positive eigenvalue $\lambda_{u}=\omega$, where $\omega>0$ is the exponent controlling the leading scaling corrections in $\mathrm{O}(2 N)$ vector models [49], and a negative eigenvalue $\lambda_{f}$, which makes the fixed point unstable. Since [38] $\lambda_{f}=-\varepsilon$ to all orders in perturbation theory, the RG dimension $y_{f}=-\lambda_{f}=\varepsilon$ of the operator that controls the crossover behavior is one in three dimensions.

\section{B. The AH field theory in the large- $N$ limit}

The existence of a stable fixed point for sufficiently large values of $N$ and, therefore, of a universality class described by the $\mathrm{AH}$ field theory is confirmed by $1 / N$ calculations [41,46-48]. Critical exponents have also been computed $[41,46]$ to order $1 / N$. For the critical exponent $v$ associated with the correlation length, one finds [41]

$$
v=1-\frac{48}{\pi^{2} N}+O\left(N^{-2}\right)
$$

for the three-dimensional model. Also the critical behavior of the two-point function

$$
G(\boldsymbol{x}, \boldsymbol{y})=\langle\operatorname{Tr} B(\boldsymbol{x}) B(\boldsymbol{y})\rangle
$$

of the gauge-invariant bilinear composite operator

$$
B_{a b}(\boldsymbol{x})=\Phi_{a}(\boldsymbol{x})^{\dagger} \Phi_{b}(\boldsymbol{x})-\frac{1}{N} \delta_{a b}|\Phi|^{2}
$$


has been considered. Here, $B_{a b}(\boldsymbol{x})$ is the coarse-grained continuum counterpart of the lattice operator $Q_{x}^{a b}$ defined in Eq. (8). At the critical point, $G(\boldsymbol{x}, \boldsymbol{y})$ has the power-law behavior

$$
\left.G(\boldsymbol{x}, \boldsymbol{y})\right|_{J=J_{c}} \sim \frac{1}{|\boldsymbol{x}-\boldsymbol{y}|^{d-2+\eta_{q}}}
$$

characterized by the critical exponent $\eta_{q}$. At order $1 / N$ one finds [46]

$$
\eta_{q}=1-\frac{32}{\pi^{2} N}+O\left(N^{-2}\right)
$$

in three dimensions.

\section{The gauge-invariant LGW framework}

A second approach that has been used to predict the critical behavior of lattice $\mathrm{AH}$ models [38,50] is the LGW framework $[49,51,52]$. It assumes that the relevant critical modes are associated with the gauge-invariant local site variable (8). As discussed in Refs. [38,50,53-55], this is a highly nontrivial assumption, as it postulates that gauge fields do not play a relevant role in the effective theory. In this approach, the order-parameter field is a traceless Hermitian matrix field $\Psi^{a b}(\boldsymbol{x})$, which can be formally defined as the average of $Q_{x}^{a b}$ over a large but finite lattice domain. The LGW field theory is obtained by considering the most general fourth-order polynomial in $\Psi$ consistent with the $\mathrm{SU}(N)$ global symmetry:

$$
\begin{aligned}
\mathcal{H}_{\mathrm{LGW}}= & \operatorname{Tr}\left(\partial_{\mu} \Psi\right)^{2}+r \operatorname{Tr} \Psi^{2} \\
& +w \operatorname{tr} \Psi^{3}+u\left(\operatorname{Tr} \Psi^{2}\right)^{2}+v \operatorname{Tr} \Psi^{4} .
\end{aligned}
$$

Also in this framework continuous transitions may only arise if the RG flow in the LGW theory has a stable fixed point.

For $N=2$, the cubic term in Eq. (20) vanishes and the two quartic terms are equivalent. Therefore, one recovers the $\mathrm{O}(3)$ symmetric vector LGW theory. Thus, for $N=2$, continuous transitions, belonging to the Heisenberg universality class, are possible. For $N \geqslant 3$, the cubic term is generically present. Its presence is usually taken as an indication that phase transitions occurring in this class of systems are generally of first order. Indeed, a straightforward mean-field analysis shows that the transition is of first order in four dimensions, where the mean-field approximation is exact. If statistical fluctuations are small-this is the basic assumption-the transition is of first order also in three dimensions. In this approach, continuous transitions may still occur, but they require a fine tuning of the microscopic parameters, leading to the effective cancellation of the cubic term [56].

It is important to note that the field-theoretical approaches based on the continuum AH field theory (1) and the effective LGW field theory (20) are not equivalent, as they make different assumptions on the role of the gauge correlations. They give different predictions, both for small and large values of $N$. For $N=2$ the continuum AH model predicts the absence of continuous transitions, due to the absence of a stable fixed point. On the other hand, a stable $\mathrm{O}(3)$ vector fixed point exists in the effective LGW theory, leaving open the possibility of observing continuous transitions.

For large values of $N$ (more precisely, for $N>N_{c}$; see Sec. III A), the continuum AH theory and the effective LGW approach give again contradictory results. If one trusts the argument based on the relevance of the cubic term, the LGW approach predicts a first-order transition unless a fine tuning of the microscopic parameters is performed. Instead, continuous transitions are possible without any fine tuning according to the continuum AH field theory. For intermediate values of $N$, that is, for $3 \leqslant N<N_{c}$, both approaches predict lattice models to undergo first-order transitions.

The field-theoretical predictions have been compared with numerical results for the lattice $\mathrm{CP}^{N-1}$ and $\mathrm{AH}$ models, loop models, and two-dimensional quantum systems [5-7,57-59]. Simulations of the $\mathrm{CP}^{1}$ model $[38,50]$ confirm the existence of continuous transitions, belonging to the $\mathrm{O}(3)$ vector universality class, for $N=2$. Therefore, the LGW theory provides the correct description of the large-scale behavior of these systems. Evidently, gauge correlations are not relevant in the $\mathrm{CP}^{1}$ model, and the continuum $\mathrm{AH}$ model does not predict the correct behavior. For $N=3$ numerical results are not yet conclusive. Indeed numerical simulations for the lattice $\mathrm{CP}^{2}$ model favor a first-order transition [50], while the results for the loop model of Refs. [57,58] apparently favor a continuous transition. The available numerical results for lattice $\mathrm{CP}^{N-1}$ models for $N \geqslant 4$ are consistent with first-order transitions [50,58-60], again confirming the LGW predictions. We note that a continuous transition has been observed in a monopole-free version of the $\mathrm{CP}^{N-1}$ model [18] for large values of $N$, demonstrating the relevance of these topological defects.

\section{THE PHASE DIAGRAM OF THE NONCOMPACT LATTICE AH MODEL}

\section{A. Transition lines and limiting cases}

To sketch the phase diagram of the noncompact lattice $\mathrm{AH}$ model with $N$-component scalar fields (see Fig. 1), it is useful to consider some particular cases in which the thermodynamic behavior is already known. No transitions are expected along the $J=0$ line, while transitions occur along the $\kappa=0$, the $J=\infty$, and the $\kappa=\infty$ lines.

Phase diagram along the $\kappa=0$ line. For $\kappa=0$ the lattice $\mathrm{AH}$ model is equivalent to a lattice formulation of the $\mathrm{CP}^{N-1}$ models with explicit lattice gauge variables [50]. Its phase diagram has been recently discussed in Refs. [50,59]. There are two phases separated by a finite-temperature transition, where the order parameter is the gauge-invariant matrix defined in Eq. (8). The available estimates of the transition point $J_{c}$ are reported in Table I. The phase transition is continuous for $N=2$, belonging to the $\mathrm{O}(3)$ vector universality class [49] (accurate estimates of the $\mathrm{O}(3)$ critical exponents can be found in Refs. [64-68]), and of first order for $N \geqslant 3$. It is very weak for $N=3$ [50], and it becomes stronger and stronger with increasing $N$ [59]. It is natural to conjecture that analogous transitions occur along the CM line for small values of $\kappa$. Thus, we expect a line of continuous transitions belonging to the $\mathrm{O}(3)$ universality class for $N=2$, and a line of first-order transitions for $N \geqslant 3$. Note that, for $N=2$, this scenario implies the stability of the $\mathrm{O}(3)$ critical behavior against perturbations due to the noncompact gauge field. We report numerical evidence that confirms it. 
TABLE I. Estimates of the critical values $J_{c}$ for $\kappa=0$ and $\kappa \rightarrow$ $\infty$. We also report the nature of the transition: $\mathrm{FO}$ and $\mathrm{O}(n)$ indicate a first-order transition and a continuous transition in the $\mathrm{O}(n)$ vector universality class, respectively. The estimates of $J_{c}$ for $\kappa \rightarrow \infty$ for $7 \leqslant N \leqslant 20$ are obtained by interpolating the results of Ref. [61] for the lattice $\mathrm{O}(n)$ vector model (the uncertainty on these interpolations is safely below 1 on the last reported digit). For $N \rightarrow \infty$, the results of Ref. [61] allow us to obtain $J_{c}(\kappa \rightarrow \infty)=b_{\infty}+b_{\infty, 1} N^{-1}+$ $O\left(N^{-2}\right)$, with $b_{\infty}=0.252731 \ldots$ and $b_{\infty, 1} \approx-0.0585$.

\begin{tabular}{lllll}
\hline \hline$N$ & \multicolumn{1}{c}{$J_{c}(\kappa=0)$} & \multicolumn{1}{c}{$J_{c}(\kappa=\infty)$} \\
\hline 2 & $0.7102(1)[50]$ & $\mathrm{O}(3)$ & $0.233965(2)[62]$ & $\mathrm{O}(4)$ \\
3 & $0.6196(2)[50]$ & FO & $0.23813(3)[63]$ & $\mathrm{O}(6)$ \\
4 & $0.5636(1)[50]$ & FO & $0.24084(1)[56]$ & $\mathrm{O}(8)$ \\
7 & $0.4714(5)[59]$ & FO & 0.244 & $\mathrm{O}(14)$ \\
10 & $0.4253(5)[59]$ & FO & 0.247 & $\mathrm{O}(20)$ \\
15 & $0.381(1)[59]$ & FO & 0.249 & $\mathrm{O}(30)$ \\
20 & $0.353[59]$ & FO & 0.250 & $\mathrm{O}(40)$ \\
$\infty$ & $0.252731 \ldots$ & FO & $0.252731 \ldots[61]$ & $\mathrm{O}(\infty)$ \\
\hline \hline
\end{tabular}

Phase diagram along the $J=\infty$ line. For $J \rightarrow \infty$ the relevant configurations are those that minimize $H_{z}$ [cf. Eq. (3)]. There is no frustration, so that we obtain

$$
\bar{z}_{x} \cdot \lambda_{x, \mu} z_{x+\hat{\mu}}=1,
$$

which implies $z_{x}=\lambda_{x, \mu} z_{x+\hat{\mu}}$. A repeated use of this relation along a plaquette implies

$$
\lambda_{x, \mu} \lambda_{x+\hat{\mu}, \nu} \bar{\lambda}_{x+\hat{\nu}, \mu} \bar{\lambda}_{x, v}=1
$$

on each plaquette. Therefore, by an appropriate gauge transformation we obtain $A_{\boldsymbol{x}, \mu}=2 \pi n_{\boldsymbol{x}, \mu}$, where $n_{\boldsymbol{x}, \mu} \in \mathbb{Z}$. We thus obtain a dual loop representation of the 3D XY model, which is expected to undergo an "inverted" $\mathrm{XY}$ transition, i.e., a transition belonging to the $\mathrm{XY}$ universality class but with inverted high- and low-temperature phases [69]. Such a transition occurs at [70] $\kappa_{c}(J=\infty)=0.076051(2)$ [we obtained it by using the estimate $\beta_{c}=3.00239(6)$ reported in Ref. [70] and identifying $\left.\kappa_{c}=\beta_{c} /\left(4 \pi^{2}\right)\right]$. The number $N$ of components does not play any role here and the critical behavior does not depend on $N$. Note that the $\mathrm{MH}$ line separates two phases in which the matter field is magnetized, as it is for $J=\infty$. Thus, along this line the field $z$ would act as a spectator and, therefore, it is natural to conjecture that the same inverted XY behavior holds on the line separating the molecular and Higgs phases for finite but large $J$.

Phase diagram along the $\kappa=\infty$ line. In the $\kappa \rightarrow \infty$ limit, we have $A_{x, \mu}=0$ apart from gauge transformations. Therefore, in this limit the $\mathrm{N}$-component AH model can be exactly mapped onto the standard real $2 \mathrm{~N}$-component vector model, which undergoes a continuous transitions for any $N$ (see Table I). At finite $\kappa$, the RG flow of the continuum AH model predicts that gauge modes are a relevant perturbation of the $\mathrm{O}(2 N)$ fixed point [see Eq. (14) and the subsequent discussion in Sec. III A]. Therefore, if the $\mathrm{CH}$ transitions are continuous, they do not belong to the $\mathrm{O}(2 N)$ vector universality class. However, the $\mathrm{O}(2 N)$ continuous transition for $\kappa=\infty$ may give rise to crossover phenomena for large values of $\kappa$.

On the basis of the above considerations, the most natural hypothesis of phase diagram is the one reported in Fig. 1, with three different phases. For small $J$ and any $\kappa$ there is a phase (it will be named the Coulomb phase) in which the $z$ fields are disordered and the gauge modes are in the inverted XY low-temperature phase; for large $J$ and large $\kappa$ (Higgs superconducting phase) there is a phase in which the $z$ fields are ordered and the gauge modes are in the inverted $\mathrm{XY}$ high-temperature phase; for large $J$ and small $\kappa$ (mixed molecular phase) gauge interactions are long ranged, while the spin degrees of freedom condense. Presumably, for any $N$ the three transition lines meet at a multicritical point at $\left(\kappa_{m c}, J_{m c}\right)$ (see Fig. 1). This phase diagram was proposed for $N=2$ in Refs. [22,23], but it should hold for any $N \geqslant 2$ (see also Ref. [71]).

For any $N$, we expect the $\mathrm{MH}$ transition line to be continuous, in the XY universality class, for any $J>J^{*}$, where $J^{*}$ may coincide with the position of the multicritical point, i.e., $J^{*} \geqslant J_{m c}$. Along this transition line the spins are expected to be frozen. They should only act as spectators.

The transitions along the $\mathrm{CM}$ line are expected to have the same nature as the $\kappa=0$ transition, at least for $\kappa<\kappa^{*}$, where $\kappa^{*}$ must satisfy $\kappa^{*} \leqslant \kappa_{m c}$. As we said, we do not expect the addition of $H_{g}$ for small $\kappa$ to change the nature of the transition. Therefore, as it occurs for $\kappa=0$, we expect gauge modes to be irrelevant. This suggests that these transitions are controlled by the LGW $\Phi^{4}$ field theory (20), in which gauge modes are effectively integrated out. Thus, they should belong to the $\mathrm{O}(3)$ vector universality class for $N=2$ and be of first order for $N \geqslant 3$. Like the transition of the $\mathrm{CP}^{N-1}$ model for $\kappa=0$, the CM transition line is characterized by the condensation of the gauge-invariant bilinear operator (8).

Finally, along the $\mathrm{CH}$ transition line, both scalar and gauge fields change their long-distance behavior. Therefore, we expect this transition line to be described by the continuum $\mathrm{AH}$ model (1), whose RG flow predicts that continuous transitions may be observed only for $N>N_{c}$ (see Sec. III A). Along the $\mathrm{CH}$ line the gauge-invariant bilinear operator (8) is expected to be an appropriate order parameter.

\section{B. Nature of the multicritical point for $N=2$}

As discussed above, the phase diagram of the noncompact lattice AH model is characterized by three transition lines meeting at a multicritical point. To discuss its nature within the field-theory framework, it is crucial to identify the relevant critical modes. For $N=2$, we expect continuous transitions along the $\mathrm{CM}$ line with an $\mathrm{O}(3)$ scalar order parameter, firstorder transitions along the $\mathrm{CH}$ line, and $\mathrm{XY}$ behavior along the MH line. Thus, the nature of the multicritical point is determined by the dynamics of two effective order-parameter fields, a three-component and a two-component scalar field, which are associated with the two continuous transition lines. This hypothesis seems quite reasonable, as the two transitions are associated with different degrees of freedom: Along the $\mathrm{CM}$ line the spin degrees of freedom associated with the bilinear $Q_{x}^{a b}$ defined in Eq. (8) condense, while gauge degrees of freedom control the transitions along the MH line.

Note that this description is not expected to be appropriate for $N \neq 2$ and, in particular, for large values of $N$. In that case, the $\mathrm{CH}$ transition line should be associated with the AH FP point, the CM line is of first order, while the $\mathrm{MH}$ 


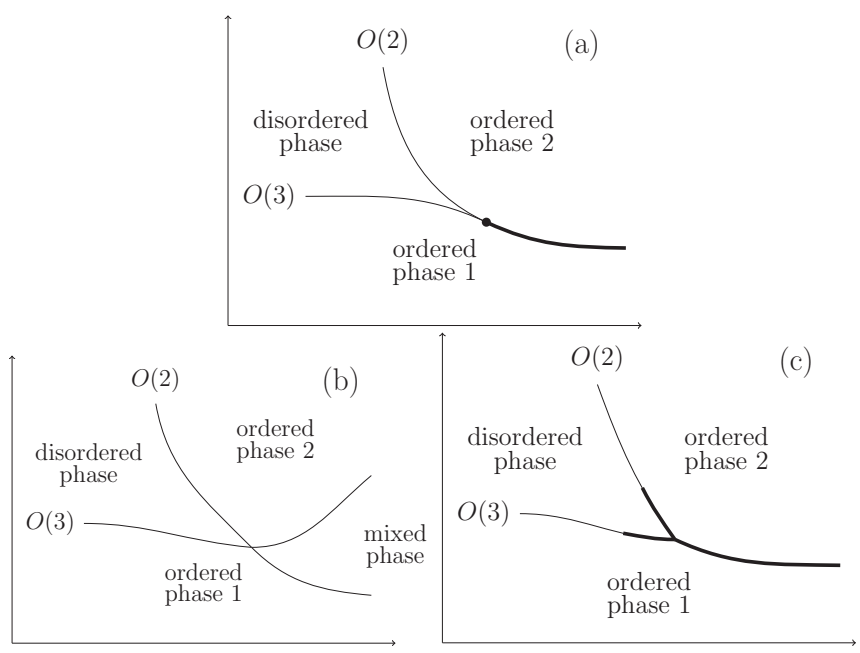

FIG. 2. Sketches of the possible phase diagrams close to a multicritical point in the plane of the two relevant variables. Thin lines represent continuous phase transitions, while thick lines represent first-order transitions. (a) Phase diagram with a bicritical point. (b) Phase diagram with a tetracritical point. (c) Phase diagram with a first-order bicritical point.

transitions belong to the XY universality class. In this case a correct description of the multicritical point should describe the interaction of the XY order parameter with the AH fields. Thus, one should consider an extension of the AH model that includes an additional two-component order parameter.

For $N=2$, assuming that the multicritical behavior effectively arises from the competition of two different scalar fields, we may investigate it within a LGW framework. If $\phi^{a}$ and $\varphi^{a}$ are a three-component and a two-component field, respectively, the most general $\Phi^{4}$ theory that is invariant under $\mathrm{O}(3) \oplus \mathrm{O}(2)$ transformations [72-75] is

$$
\begin{aligned}
\mathcal{H}= & \frac{1}{2}\left[\left(\partial_{\mu} \phi\right)^{2}+\left(\partial_{\mu} \varphi\right)^{2}\right]+\frac{1}{2}\left(r_{\phi} \phi^{2}+r_{\varphi} \varphi^{2}\right) \\
& +\frac{1}{4 !}\left[u_{\phi}\left(\phi^{2}\right)^{2}+u_{\varphi}\left(\varphi^{2}\right)^{2}+2 w \phi^{2} \varphi^{2}\right]
\end{aligned}
$$

where $\phi^{2} \equiv \sum_{a=1}^{3} \phi_{a}^{2}$ and $\varphi^{2} \equiv \sum_{a=1}^{2} \varphi_{a}^{2}$. Such a model appears in several other contexts; for instance, it is relevant for the behavior of high-temperature superconductors (see Ref. [76] and references therein). It has been extensively studied in Refs. [72-76]. Note that we are assuming here that the $\mathrm{XY}$ transition is associated with a local order parameter. In our model, this local quantity appears in disguise. Indeed, at the transition for $J=\infty$ the order parameter is nonlocal, but, because of duality, it is dual to a local quantity defined in the $\mathrm{XY}$ model. We assume that the same is true along the MH line, i.e., that one can define a nonlocal quantity that is related by duality to a local quantity. The parameter $\phi$ is related to this local order parameter, which is the one that drives the standard XY critical behavior.

In the mean-field approximation [72-74], two possible phase diagrams are possible: A phase diagram where two continuous transition lines meet a first-order transition line [see Fig. 2(a)] - the corresponding multicritical point is called bicritical-and a phase diagram where four transition lines meet [see Fig. 2(b)] — in which case the multicritical point is called tetracritical. In our case, the multicritical point is bicritical, the first-order transition line being identified with the $\mathrm{CH}$ transition line. Indeed, the numerical results reported in Refs. [22,23,30] confirm that the transitions along the $\mathrm{CH}$ line are of first order, at least for $\kappa$ not too large (they apparently disagree only far from the multicritical point). To clarify whether the bicritical transition is continuous or of first order, it is necessary to analyze the RG flow of the multicritical $\Phi^{4}$ theory (23): The multicritical transition can be continuous only if a stable fixed point can be associated with the bicritical point.

The analysis reported in Refs. [75,76] indicates that the only stable fixed point is the decoupled fixed point describing decoupled $\mathrm{O}(3)$ and $\mathrm{O}(2)$ critical behaviors, which is naturally associated with a tetracritical point [see Fig. 2(b)]. There is no stable fixed point that can be associated with a bicritical point. Indeed, fixed points that can be associated with a bicritical behavior, for instance, the $\mathrm{O}(5)$ fixed point (in this case there would be an enlargement of the symmetry at the multicritical point) and the so-called biconical fixed point, are both unstable $[75,76]$. In the absence of stable fixed points that may be associated with a bicritical point, close to the multicritical point the transitions are expected to be of first order along all three lines [see Fig. 2(c)]. Therefore, the continuous $\mathrm{O}(3)$ and XY transition lines starting from the $\kappa=0$ and $J=\infty$ lines are expected to turn into first-order transition lines before reaching the multicritical point.

The above LGW RG analysis predicts the $\mathrm{CH}$ transition line to be of first order close to the multicritical point. Increasing $\kappa$ along this line, the first-order transition should become weaker and weaker (the latent decreases) as the $\mathrm{O}(4)$ continuous transition at $\kappa=\infty$ is approached, with substantial crossover phenomena occurring for large values of $\kappa$. Alternatively, the first-order transition could turn into a continuous transition line belonging to a different universality class already for finite values of $\kappa$. We note that the existence of a corresponding universality class is still controversial (see, e.g., Refs. [7-10,12,18,19,22,23,36]).

\section{NUMERICAL RESULTS FOR THE NONCOMPACT AH MODELS}

\section{A. Simulation details and boundary conditions}

We now present a Monte Carlo (MC) study of the phase diagram of the noncompact lattice $\mathrm{AH}$ model, for $N=2,4$, 10,15 , and 25 .

As already noted, due to the peculiarities of the noncompact formulation, we cannot consider finite systems with periodic boundary conditions. Indeed, in this case there are gauge-invariant zero modes, that make the model always ill defined. The zero modes correspond to the so-called noncompact Polyakov lines, the gauge-invariant sum of the fields $A_{\boldsymbol{x}, \mu}$ along nontrivial paths winding around the lattice. Therefore, even if a maximal gauge fixing is added, the partition function is still infinite. Under these conditions, it is not clear whether finite-size scaling (FSS) methods can be safely used to investigate the critical behavior of the model. To overcome this problem, we adopt $C^{*}$ boundary conditions $[39,40]$. They preserve gauge invariance, providing a rigorous definition of the partition function in a finite volume. Moreover, they 
essentially preserve translational invariance. A detailed description is provided in the Appendix.

To update the fields $A_{x, \mu}$ and $z_{x}$ we used a Metropolis algorithm constructed as follows: The new trial field $A_{\boldsymbol{x}, \mu}$ was constructed by adding to the old one a random number in the range $\left[-\epsilon_{1}, \epsilon_{1}\right]$, while the trial field $z_{x}$ was obtained by multiplying the old field by a random unitary matrix having only a $2 \times 2$ nontrivial diagonal block (whose distance from the identity was controlled by a second parameter $\epsilon_{2}$ ). The parameters $\epsilon_{1}, \epsilon_{2}$ have been fixed in order to have an acceptance probability around $30 \%$. Since the action is linear in $z_{x}$, it is simple to write an overrelaxation algorithm which is just the complex analogue of the one typically used for $\mathrm{O}(\mathrm{N})$ models.

In order to reduce autocorrelations we combined Metropolis and overrelaxation moves in a ratio of 1:5. The estimated autocorrelation time was at most of the order of $10^{4}$ updates for the larger lattices and close to the critical point. In all the cases, i.e., for all the lattices, simulation points, and observables, our statistics consisted of $10^{3}$ or more independent thermalized samples. Final errors have been estimated using a standard self-consistent blocking and jackknife procedure.

\section{B. Observables and finite-size scaling analyses}

We compute the energy density and the specific heat, defined as

$$
E=\frac{1}{V}\langle H\rangle, \quad C=\frac{1}{V}\left(\left\langle H^{2}\right\rangle-\langle H\rangle^{2}\right),
$$

where $V=L^{3}$. We consider the two-point correlation function of the gauge-invariant operator $Q_{x}^{a b}$ defined in Eq. (8),

$$
G(\boldsymbol{x}-\boldsymbol{y})=\left\langle\operatorname{Tr} Q_{x} Q_{y}\right\rangle,
$$

where the translation invariance of the system has been taken into account (note that $Q_{x}$ is periodic also in the presence of $C^{*}$ boundary conditions; see the Appendix). The susceptibility and the (second-moment) correlation length are defined by the relations

$$
\begin{gathered}
\chi=\sum_{\boldsymbol{x}} G(\boldsymbol{x})=\widetilde{G}(\mathbf{0}), \\
\xi^{2} \equiv \frac{1}{4 \sin ^{2}(\pi / L)} \frac{\widetilde{G}(\mathbf{0})-\widetilde{G}\left(\boldsymbol{p}_{m}\right)}{\widetilde{G}\left(\boldsymbol{p}_{m}\right)},
\end{gathered}
$$

where $\widetilde{G}(\boldsymbol{p})=\sum_{x} e^{i \boldsymbol{p} \cdot \boldsymbol{x}} G(\boldsymbol{x})$ is the Fourier transform of $G(\boldsymbol{x})$, and $\boldsymbol{p}_{m}=(2 \pi / L, 0,0)$.

It is convenient to introduce RG-invariant quantities, such as the Binder parameter

$$
U=\frac{\left\langle\mu_{2}^{2}\right\rangle}{\left\langle\mu_{2}\right\rangle^{2}}, \quad \mu_{2}=\frac{1}{V^{2}} \sum_{x, y} \operatorname{Tr} Q_{x} Q_{y},
$$

and

$$
R_{\xi}=\xi / L .
$$

At continuous phase transitions they are expected to scale as [49] (we denote by $R$ a generic RG invariant quantity)

$$
R(\beta, L) \approx f_{R}(X), \quad X=\left(\beta-\beta_{c}\right) L^{1 / \nu},
$$

where $v$ is the critical exponent associated with the correlation length, and $\beta$ is the parameter we vary in the system (in

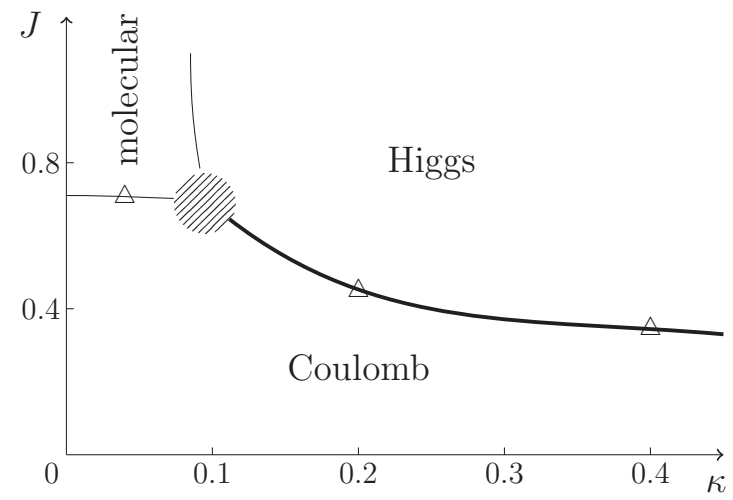

FIG. 3. Phase diagram of the noncompact $N=2$ lattice AH model, as obtained from the data of Refs. [22,23]. Triangles correspond to the transition points obtained in the present work. The shadowed blob indicates the region where the transition lines meet and the transitions along the three lines are predicted to be of first order (see Sec. IV B).

the following sections $J$ will play the role of $\beta$ ). Scaling corrections decaying as $L^{-\omega}$ have been neglected in Eq. (30), where $\omega$ is the exponent associated with the leading irrelevant operator. The function $f_{R}(X)$ is universal up to a multiplicative rescaling of its argument. In particular, $U^{*} \equiv f_{U}(0)$ and $R_{\xi}^{*} \equiv f_{R_{\xi}}(0)$ are universal, depending only on the boundary conditions and the aspect ratio of the lattice. Since $R_{\xi}$ defined in Eq. (29) is an increasing function of $\beta$, we can write

$$
U(\beta, L)=F_{U}\left(R_{\xi}\right)+O\left(L^{-\omega}\right),
$$

where $F_{U}$ now depends on the universality class, boundary conditions, and lattice shape, without any nonuniversal multiplicative factor. The scaling relation (31), which does not involve any nonuniversal parameter, is particularly convenient to test universality-class predictions and to identify weak firstorder transitions [38,50].

At first-order transitions the probability distributions of the energy and of the magnetization are expected to show a double peak for large values of $L$. However, in order to definitely identify a first-order transition, it is necessary to perform a more careful analysis of the large- $L$ scaling behavior of the distributions or, equivalently, of the specific heat and of the Binder cumulants [77-81]. Regarding the specific heat $C$, for each lattice size $L$, there exists a value $\beta_{\max , C}(L)$ of $\beta$ where $C$ takes its maximum value $C_{\max }(L)$. For large volumes, we have [77]

$$
\begin{gathered}
C_{\max }(L)=\frac{V}{4} \Delta_{h}^{2}+O(1), \\
\beta_{\max , C}(L)-\beta_{c} \approx c V^{-1},
\end{gathered}
$$

where $\Delta_{h}=E_{+}-E_{-}$is the latent heat, and $E_{+}$and $E_{-}$are the values of the energy corresponding to the two maxima of the energy-density distribution.

As discussed in Refs. $[38,50]$ the Binder parameter is a particularly convenient quantity to identify first-order transitions. In this case [78], for each $L, U(\beta, L)$ has a maximum $U_{\max }(L)$ at $\beta=\beta_{\max , U}(L)<\beta_{c}$ which scales, for sufficiently 

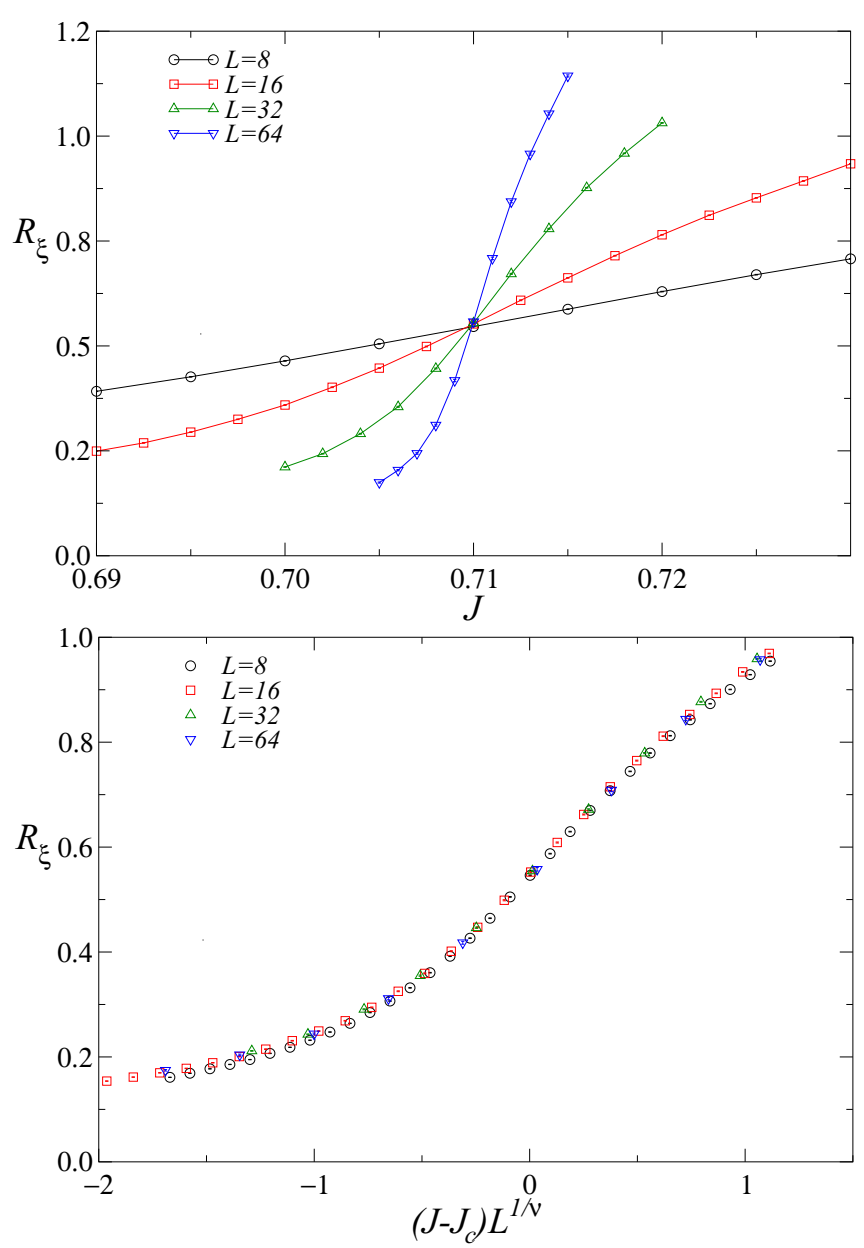

FIG. 4. Plot of $R_{\xi}$ for $N=2$ and $\kappa=0.04$ (along the CM transition line) for lattice sizes up to $L=64$. Top: $R_{\xi}$ versus $J$; data show a crossing point at $J=J_{c}=0.7099(1)$. Bottom: $R_{\xi}$ versus $\left(J-J_{c}\right) L^{1 / v}$ using the $\mathrm{O}(3)$ critical exponent $v=0.7117$ [64-67].

large values of $L$, as

$$
\begin{gathered}
U_{\max } \sim a V+O(1), \\
\beta_{\max , U}(L)-\beta_{c} \approx b V^{-1} .
\end{gathered}
$$

This should be contrasted with the behavior at a continuous transition, where $U$ is always finite. Thus, we can distinguish first-order from continuous transitions by looking at the behavior of $U$ as $L$ increases. In particular, the absence of scaling when plotting the data of $U$ versus $R_{\xi}$ may be considered as an evidence in favor of a first-order transition.

Note that FSS also holds at first-order transitions [80,82-84], although it is more sensitive to the geometry and to the nature of the boundary conditions [81]; for instance, FSS differs for boundary conditions that favor or do not favor the different phases coexisting at the transition $[85,86]$. In the case of 3D cubic systems with boundary conditions respecting translation invariance, such as periodic boundary conditions, FSS is typically characterized by an effective exponent $v=1 / d=1 / 3$, so that $\alpha / v=d=3$. Thus, effective exponents that decrease towards $1 / 3$ as $L$ increases, signal a discontinuous transition.

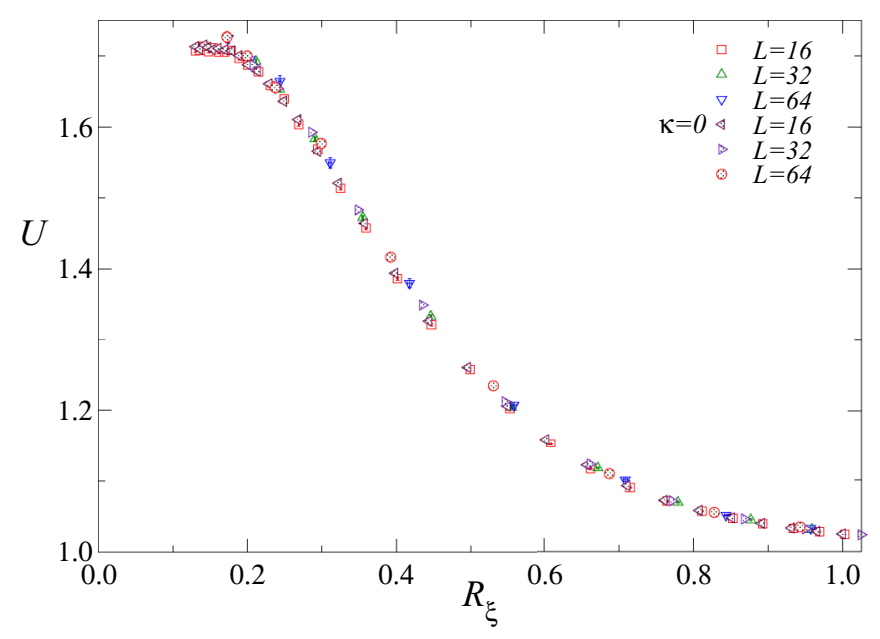

FIG. 5. Plot of $U$ versus $R_{\xi}$ for the noncompact $N=2$ lattice $\mathrm{AH}$ model at $\kappa=0.04$ and $\kappa=0$ (along the CM transition line). All data fall onto a single curve, providing a robust evidence that the transition belongs to the $\mathrm{O}(3)$ universality class.

\section{The noncompact lattice $\mathrm{AH}$ model at $N=2$}

The lattice AH model with $N=2$ has already been studied in Refs. [22,23,30], obtaining the phase diagram shown in Fig. 3. We present a different FSS analysis, using $C^{*}$ boundary conditions, which is not affected by the pathologies of the periodic boundary conditions (see the Appendix). In particular, we present results along the $\mathrm{CM}$ and $\mathrm{CH}$ transition lines (see Fig. 3).

To begin with, we discuss the critical behavior along the CM line starting at $\kappa=0, J=J_{c}=0.7102(1)$. We present a FSS analysis of MC data taken at fixed $\kappa=0.04$. The data of $R_{\xi}$ (see Fig. 4) show a crossing point at $J_{c}=0.7099(1)$, which is very close to the critical value at $\kappa=0$. The plot of the Binder parameter $U$ versus $R_{\xi}$ (see Fig. 5) shows that the critical behavior is the same for $\kappa=0$ and $\kappa=0.04$. Since the $\mathrm{CP}^{1}$ transition belongs to the $\mathrm{O}(3)$ vector universality class, the same is expected for the transition at $\kappa=0.04$. The $\mathrm{O}(3)$ scaling is also confirmed by the scaling of $R_{\xi}$ and of the susceptibility: This is observed when plotting $R_{\xi}$ versus $\left(J-J_{c}\right) L^{1 / v}$ and the ratio $\chi / L^{2-\eta_{q}}$ versus $R_{\xi}$ using the $\mathrm{O}(3)$ critical exponents $v \approx 0.7117$ and $\eta_{q} \approx 0.0378$ [64-67] (see Figs. 4 and 6).

To investigate the nature of the transitions along the $\mathrm{CH}$ line, we have performed MC simulations at $\kappa=0.2$ and $\kappa=0.4$. As we will see, in both cases the data clearly favor a first-order transition. This confirms the analysis reported in Refs. [23,30]. It disagrees instead with Ref. [22], which claimed the transition for $\kappa=0.4$ to be continuous. Figure 7 reports the behavior of the Binder parameter $U$ for $\kappa=0.2$ and 0.4: Data indicate the presence of a transition at $J=$ $J_{c} \approx 0.472$ for $\kappa=0.2$ and at $J=J_{c} \approx 0.353$ for $\kappa=0.4$, the latter result being in agreement with Ref. [22]. In Fig. 8 we plot $U$ versus $R_{\xi}$ for both $\kappa=0.2$ and 0.4. Data do not scale, providing evidence in favor of a weak first-order transition for both $\kappa=0.2$ and 0.4 (see the discussion in Sec. V B). Comparing the data, we observe that the first-order transition becomes weaker with increasing $\kappa$, in agreement with the expectation that the latent heat vanishes in the limit $\kappa \rightarrow \infty$. 


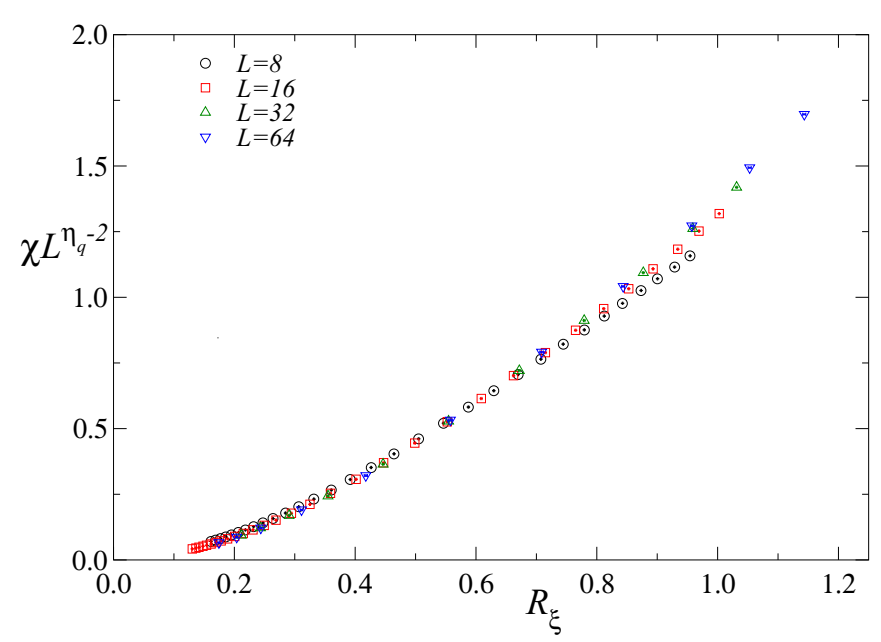

FIG. 6. Plot of $\chi / L^{2-\eta_{q}}$ at $\kappa=0.04$ versus $R_{\xi}$ along the CM transition line, using the $\mathrm{O}(3)$ value $\eta_{q}=0.0378$ [64-67]. Results for the $N=2 \mathrm{AH}$ model.

For comparison we also report data for the $\mathrm{O}(4)$ vector model (for which $U$ and $R_{\xi}$ are obtained from the spin-2 correlation function of the vector model) with the $C^{*}$ boundary con-
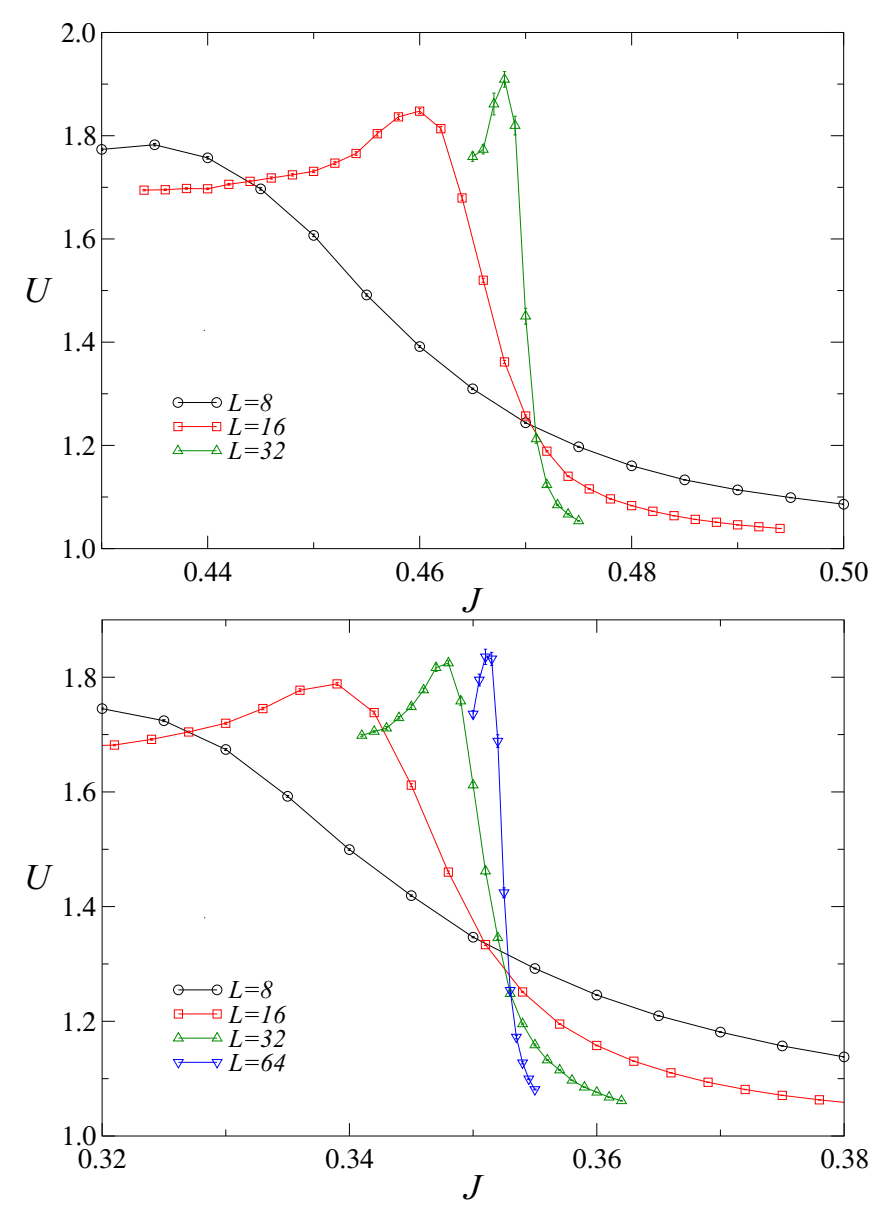

FIG. 7. Binder parameter $U$ for the $N=2$ lattice AH model, at $\kappa=0.2$ and for $L \leqslant 32$ (top) and at $\kappa=0.4$ for $L \leqslant 64$ (bottom). Both transitions should be along the $\mathrm{CH}$ transition line.
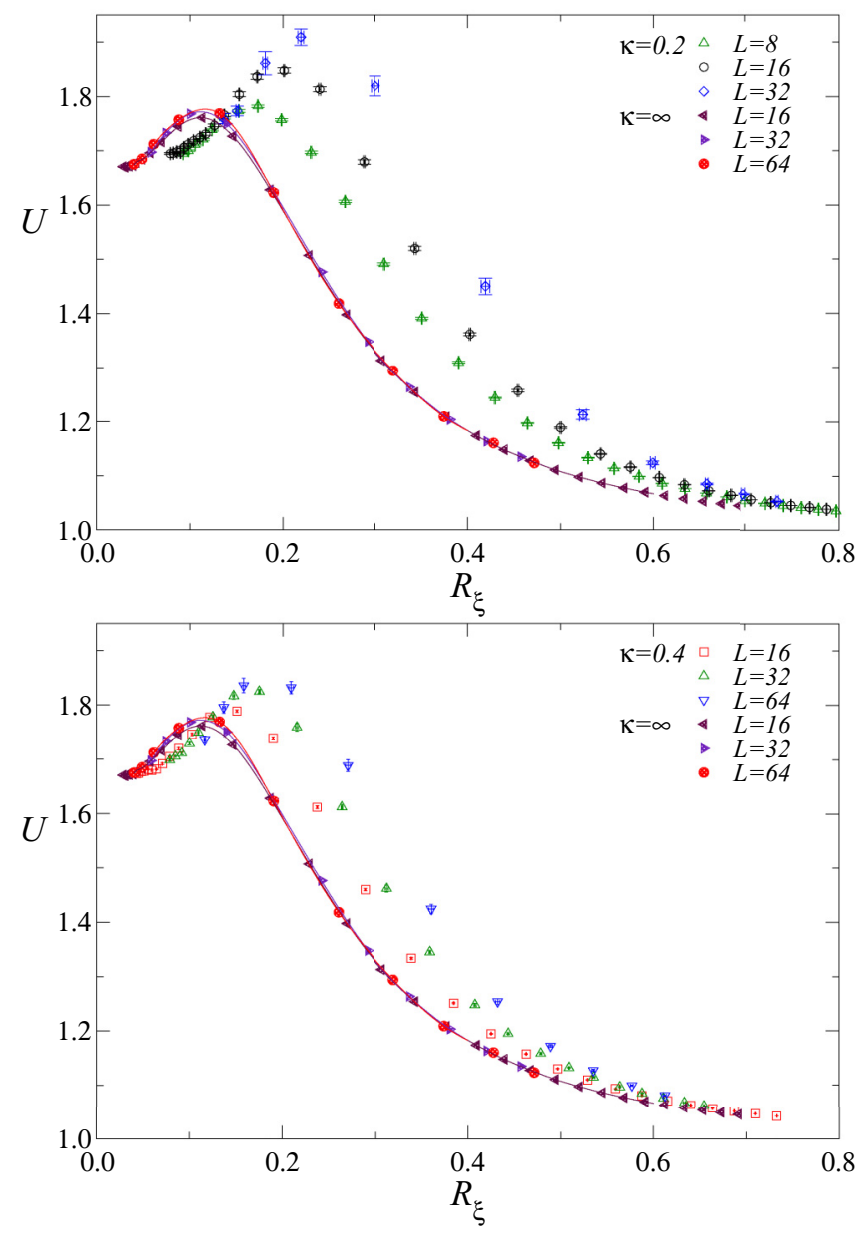

FIG. 8. Estimates of $U$ versus $R_{\xi}$ for the $N=2 \mathrm{AH}$ model at $\kappa=$ 0.2 (top) and $\kappa=0.4$ (bottom), thus along the $\mathrm{CH}$ transition line. For comparison, $O(4)$ data, corresponding to $\kappa=\infty$, are also reported; the continuous lines are cubic spline interpolations and have the only purpose of guiding the eye.

ditions, to identify possible crossover effects, which indeed seem to appear.

\section{The noncompact lattice AH model at large $N$}

We now present numerical results for large values of $N$. As discussed in Sec. IV, we expect a phase diagram characterized by three phases also for $N>2$. However, for $N \neq 2$, the CM transition line is expected to be of first order, as for $\kappa=0$. On the other hand, continuous transitions may appear along the $\mathrm{CH}$ transition line for sufficiently large $N$, since the RG flow of the continuum AH theory has a stable fixed point (see Sec. II).

We first consider the $N=25$ model, performing simulations for $\kappa=0.4$. The data of $R_{\xi}$ (see Fig. 9) show a crossing point for $J_{c} \approx 0.295$, which we identify as a transition point along the $\mathrm{CH}$ line. To understand the order of the transition, we plot the Binder parameter $U$ versus $R_{\xi}$ (see Fig. 10). Data scale nicely, strongly suggesting that the transition is continuous. To determine the critical exponent $v$ and obtain a more accurate estimate of the critical point, we fit $U$ 


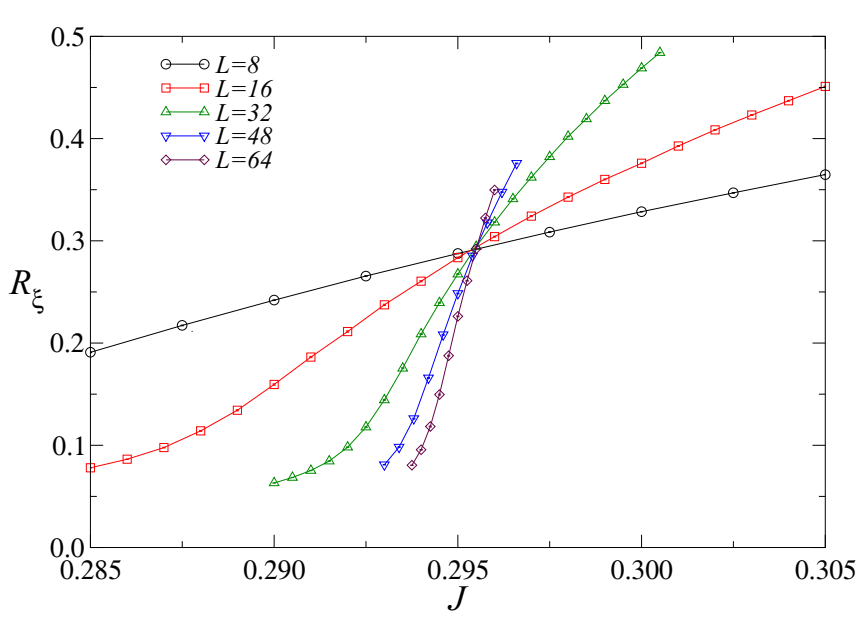

FIG. 9. Estimates of $R_{\xi} \equiv \xi / L$ for the $N=25$ lattice AH model at $\kappa=0.4$, for several lattice sizes up to $L=64$. The data show clearly a crossing point providing an estimate of the critical value $J_{c} \approx 0.2955$.

and $R_{\xi}$ to

$$
R(J, L)=f_{R}(X), \quad X=\left(J-J_{c}\right) L^{1 / v},
$$

using a polynomial approximation for $f_{R}(X)$. To estimate the role of the scaling corrections we restrict the fit to the data satisfying $L \geqslant L_{\min }$, with $L_{\min }=16,32$. For $L_{\min }=$ 16 we obtain $v=0.789(2), v=0.785(1)$ from the analysis of $U$ and $R_{\xi}$, respectively. For $L_{\min }=32$, we find $v=$ $0.782(5), 0.796(2)$. The variation of the results appears larger than the statistical errors, indicating that scaling corrections are significant, at the level of precision of our data. We have thus performed fits that include scaling corrections, fitting the data to

$$
R(J, L)=f_{R}(X)+L^{-\omega} g_{R}(X),
$$

using a polynomial approximation for both scaling functions. To improve the accuracy of the estimates, we have performed

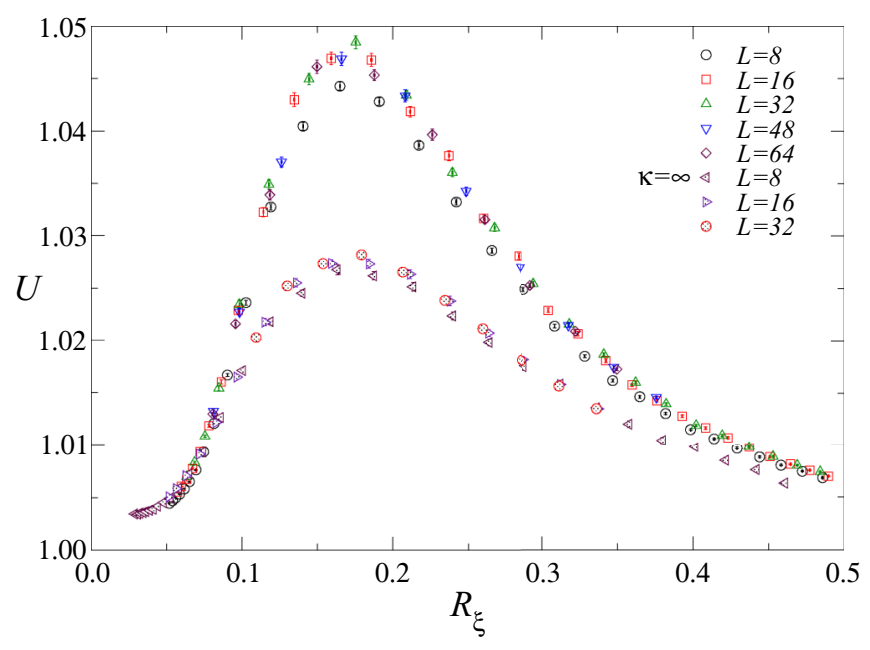

FIG. 10. Plot of $U$ versus $R_{\xi}$ for the $N=25$ lattice AH model at $\kappa=0.4$. For comparison we also report data for the $\mathrm{O}(50)$ vector model, corresponding to the $\kappa \rightarrow \infty$ limit.

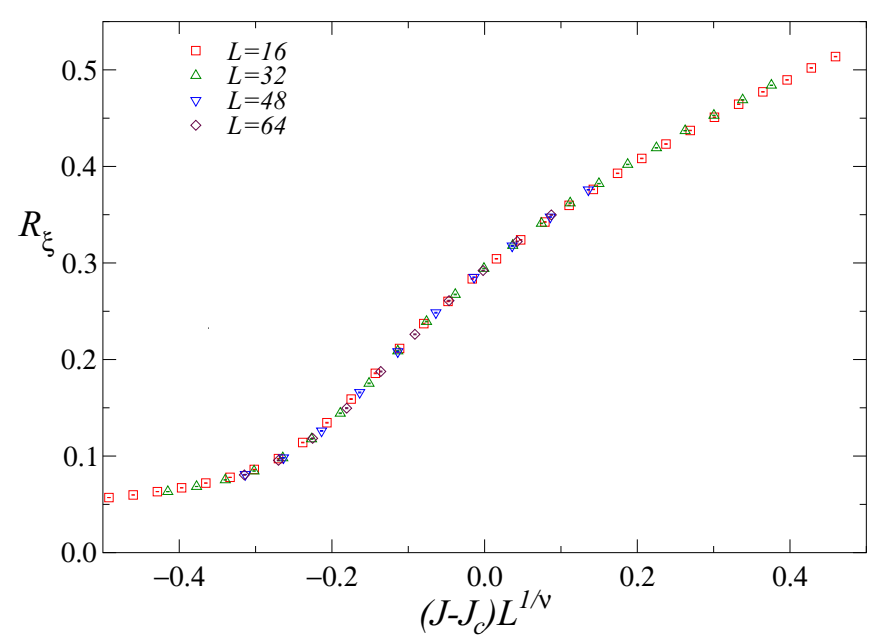

FIG. 11. Plot of $R_{\xi} \equiv \xi / L$ versus $\left(J-J_{c}\right) L^{1 / v}$ at at $\kappa=0.4$ for $N=25$. We use $J_{c}=0.295511$ and $v=0.802$.

a combined fit of the two observables. It turns out that our data are not precise enough to allow us to determine the exponent $\omega$. The $\chi^{2}$ of the fit takes essentially the same value for any $\omega \gtrsim 0.8$. Correspondingly, $v$ varies between 0.796 and 0.808 , with a statistical error of 0.002 at fixed $\omega$. This analysis allows us to estimate $v=0.802(8)$. The quality of the fit is excellent, as can be seen from Fig. 11 . The estimate of $v$ is very different from that corresponding to the $\mathrm{O}(50)$ vector model, $v \approx 0.96$ (we use here the large- $N$ expansion of $v$ ), confirming the instability of the $\mathrm{O}(50)$ fixed point in the AH field theory. This is also confirmed by the comparison of the plots of $U$ versus $R_{\xi}$ (see Fig. 10), where we report data for the $\mathrm{O}(50)$ vector model, i.e., $U$ and $R_{\xi}$ as obtained from the spin-2 correlation function in the vector model.

The estimate of $v$ is very close to the estimate obtained using the $1 / N$ expansion at order $1 / N[41,46-48], v_{l n}=0.805$ [see Eq. (15)]. The large- $N$ expansion appears to be very accurate for $v$ at $N=25$. It is interesting to note that the exponent $\omega$ is equal to 1 for $N=\infty$ and thus we expect it to be close to 1 also for $N=25$. Using this information, we can verify that the large- $N$ expansion is probably accurate for the exponent $v$ at the level of a few per thousand. Indeed, our combined fits give $v=0.805(2)$ and $v=0.808(2)$ for $\omega=1$ and 0.8 , respectively.

The analysis also provides estimates of $J_{c}$ and of the universal quantities $R_{\xi}^{*}$ and $U^{*}$, the last two quantities being the asymptotic values $(L \rightarrow \infty)$ of $R_{\xi}$ and $U$ computed for $J=J_{c}$. The results are reported in Table II. We have also analyzed the susceptibility $\chi$ in order to determine the exponent $\eta_{q}$. We have performed fits to $\chi=L^{2-\eta_{q}} f_{\chi}\left(R_{\xi}\right)$, which has the advantage that neither $J_{c}$ nor $v$ appear in the fitting function. We obtain $\eta_{q}=0.923(1)$ and $\eta_{q}=0.901(1)$, if we only consider the data with $L \geqslant 16$ and 32, respectively. There are clearly scaling corrections. We have therefore performed fits to

$$
\chi=L^{2-\eta_{q}}\left[f_{\chi}\left(R_{\xi}\right)+L^{-\omega} g_{\chi}\left(R_{\xi}\right)\right],
$$

where we use polynomial approximations for $f_{\chi}\left(R_{\xi}\right)$ and $g_{\chi}\left(R_{\xi}\right)$. The $\chi^{2}$ of the fit has a shallow minimum for $0.9 \lesssim \omega \lesssim 1.5$. As $\omega$ varies in this interval, $\eta_{q}$ varies from 
TABLE II. We report the estimates of the critical coupling $J_{c}$, of the critical exponents $v$ and $\eta_{q}$, and of the universal critical values $R_{\xi}^{*}$ and $U^{*}$ for $C^{*}$ boundary conditions at the continuous transitions observed for $N=10,15$, and 25 along the $\mathrm{CH}$ transition line $(\kappa=0.4)$. We also report the estimates $\nu_{\ln }$ and $\eta_{q, \text { ln }}$ obtained from the $O(1 / N)$ approximations reported in Eqs. (15) and (19).

\begin{tabular}{|c|c|c|c|c|c|c|c|}
\hline$N$ & $J_{c}$ & $v$ & $v_{\text {ln }}$ & $\eta_{q}$ & $\eta_{q, \ln }$ & $R_{\xi}^{*}$ & $U^{*}$ \\
\hline 25 & $0.295511(4)$ & $0.802(8)$ & 0.805 & $0.883(7)$ & 0.870 & $0.29405(5)$ & $1.0254(1)$ \\
\hline 15 & $0.309798(6)$ & $0.721(3)$ & 0.676 & $0.815(10)$ & 0.784 & $0.316(1)$ & $1.0433(3)$ \\
\hline
\end{tabular}

$0.880(5)$ and $0.887(3)$. We thus end up with the estimate $\eta_{q}=0.883(7)$. The corresponding scaling plot is shown in Fig. 12: Data scale quite precisely onto a single curve for $L \geqslant 32$. Note that the final estimate is essentially consistent with the large- $N$ result $\eta_{q, \text { ln }}=0.870$ [see Eq. (19)].

We have also observed a transition for $N=15$ along the line $\kappa=0.4$. Indeed, data for $R_{\xi}$ and $U$ show a crossing point for $J_{c} \approx 0.31$. To identify the order of the transition, we plot $U$ as a function of $R_{\xi}$ (see Fig. 13). Scaling corrections are clearly visible, but note that the data for $L \geqslant 32$ lie on top of each other. The Binder parameter does not increase with the size, indicating that the transition is continuous. To estimate the critical exponents, we have repeated the analysis we did for $N=25$. Scaling corrections are significant, as it appears from Fig. 13. Therefore, they must be taken into account to obtain reliable estimates. We perform combined fits of $R_{\xi}$ and $U$ to the ansatz (37). If we only include data with $L \geqslant 16$, the $\chi^{2}$ of the fit is essentially constant for $1 \lesssim \omega \lesssim 2$. In this range of values of $\omega$, the exponent $v$ varies between $0.722(2)$ and $0.720(2)$, allowing us to obtain the final estimate $0.721(3)$. Results for $J_{c}$ and for the critical values $R_{\xi}^{*}$ and $U$ are collected in Table II. The corresponding scaling plot is reported in Fig. 14: The scaling behavior is excellent. We have also determined the exponent $\eta_{q}$, fitting $\chi$ to Eq. (38). We obtain $\eta_{q}=0.815(10)$. Again we can compare the results for $v$ and $\eta_{q}$ with the large- $N$ results. In this case (see Table II), some discrepancies are observed, indicating that for $N=15$ the corrections of order $1 / N^{2}$ are now significant. It is worth noting that the deviations are substantially consistent with the

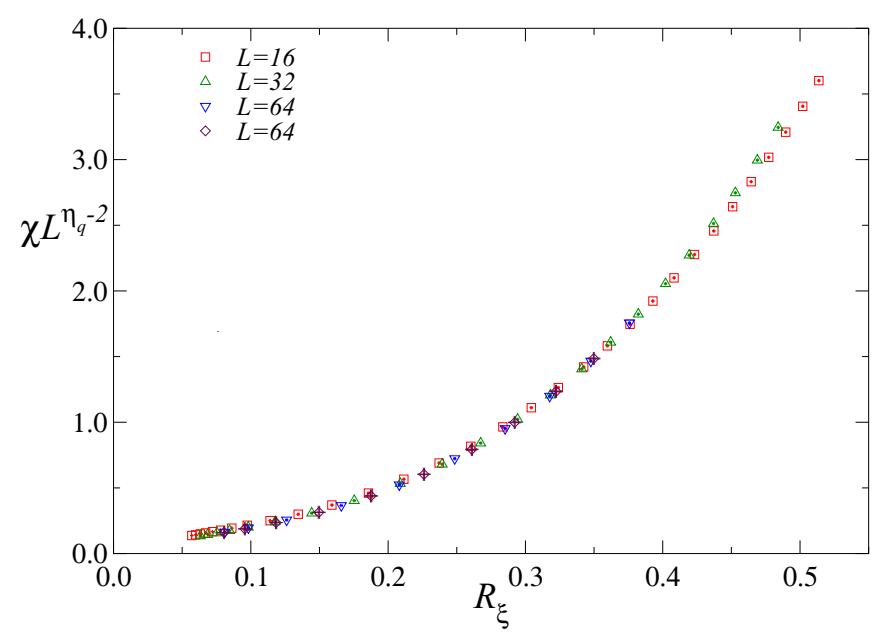

FIG. 12. Plot of $\chi L^{\eta_{q}-2}$ versus $R_{\xi}$ for the $N=25$ lattice $\mathrm{AH}$ model at $\kappa=0.4$. We use $\eta_{q}=0.883$. presence of $1 / N^{2}$ corrections, with coefficients of the size of those of the $1 / N$ terms, for both $v$ and $\eta_{q}$.

\section{E. The noncompact lattice AH model for intermediate values of $N$}

In the previous sections, we observed that the $\mathrm{CH}$ transition line is of first order for $N=2$, while it is continuous for $N=$ 15 and 25. Therefore, there must be an intermediate number $N_{\ell}$, where the nature of the transition changes: For $N \geqslant N_{\ell}$ the transition is continuous, while for $N<N_{\ell}$ it is of first order. An analogous behavior is predicted by the continuum $\mathrm{AH}$ field theory, as discussed in Sec. III A. The results of the previous sections give $2<N_{\ell}<15$. In this section we present some results for $N=4$ and $N=10$, that further constrain $N_{\ell}$.

In Fig. 15 we plot $U$ versus $R_{\xi}$ for $N=4$ along the line $\kappa=0.4$. As it occurs for $N=2$ (see Sec. V C), the data do not scale. Moreover, the Binder parameter $U$ has a maximum that increases with $L$. The data therefore favor a first-order transition, allowing us to conclude $N_{\ell}>4$. Note that the increase of the maximum of $U$ with the size signals that the first-order transition is stronger than for $N=2$. The transition is, however, too weak to allow us to reliably estimate the latent heat using lattice sizes up to $L=64$. This is a serious obstruction to what would be the natural strategy to estimate $N_{\ell}$, i.e., to determine the behavior of the latent heat as a function of $N$.

To obtain an upper bound on $N_{\ell}$, we performed simulations for $N=10$ at $\kappa=0.4$. Estimates of $U$ against $R_{\xi}$ are shown in Fig. 15. The maximum of the Binder parameter appears to

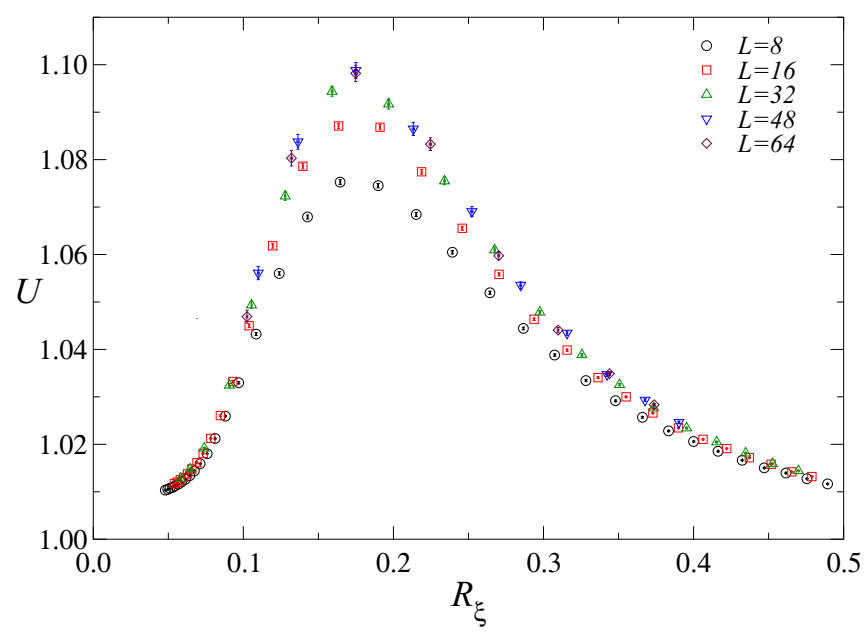

FIG. 13. Plot of $U$ versus $R_{\xi}$ for the $N=15$ lattice $\mathrm{AH}$ model at $\kappa=0.4$. 

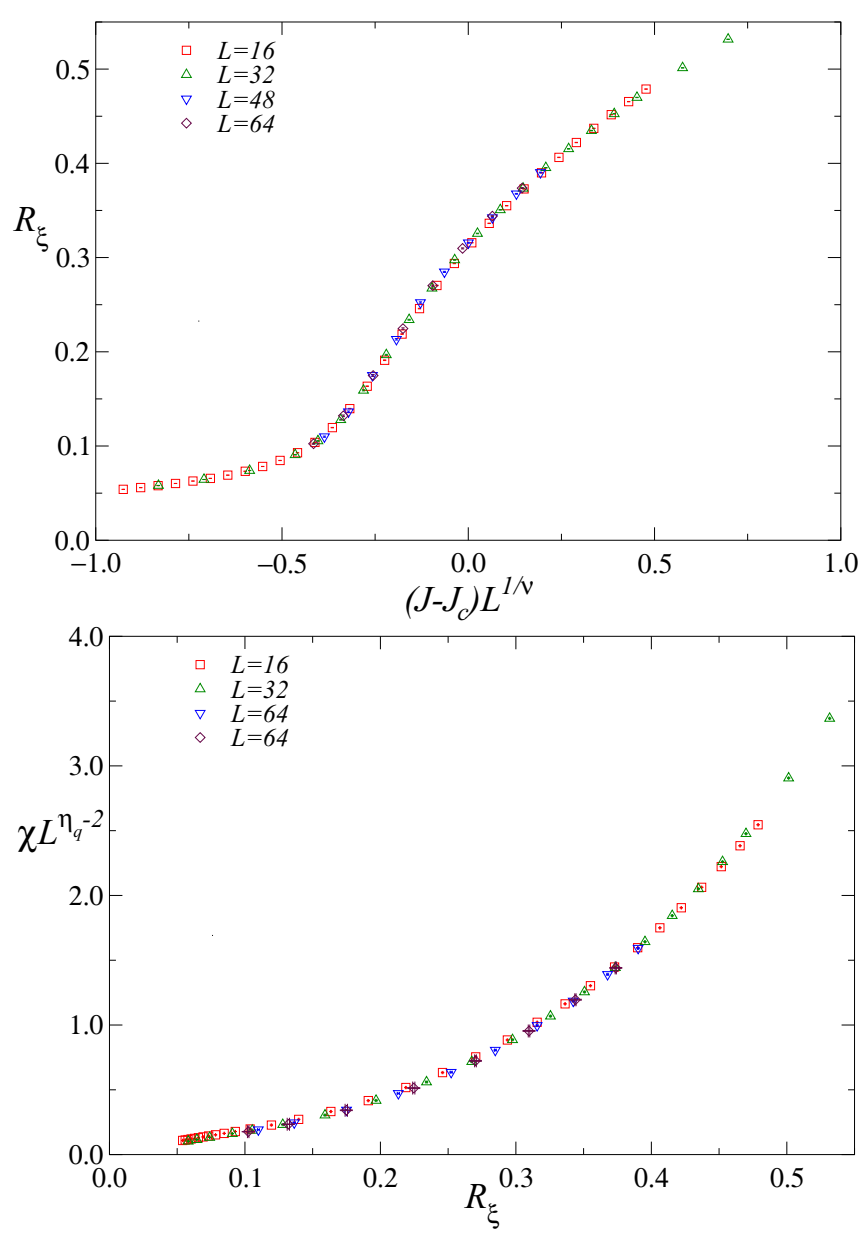

FIG. 14. Top: $R_{\xi} \equiv \xi / L$ versus $\left(J-J_{c}\right) L^{1 / v}$ for $J_{c}=0.309798$ and $v=0.721$. Bottom: $L^{-2+\eta_{q}} \chi$ versus $R_{\xi}$ for $\eta_{q}=0.815$. Results for the $N=15$ lattice $\mathrm{AH}$ model at $\kappa=0.4$ for several lattice sizes up to $L=64$.

increase with the size for small values of $L$, but the results for $L=64$ and $L=48$ apparently fall one on top of the other. Therefore, data suggest that the transition is continuous, implying the upper bound $N_{\ell}<10$.

To determine the critical exponent $v$ for $N=10$, we perform a combined fit of $U$ and $R_{\xi}$ to Eq. (37). In this case the fit is sensitive to $\omega$ and indeed the fit gives $\omega=1.05(10)$ and $v=0.642(4)$. However, the $\chi^{2}$ per degree of freedom (DOF) is quite large, $\chi^{2} / \mathrm{DOF} \approx 16$. Clearly, there are still significant scaling corrections that are not taken into account by the scaling ansatz (37). Therefore, the statistical errors are not reliable: Systematic errors due to the neglected scaling corrections are significantly larger. To get a rough idea of the size of the systematic errors, we can compare the previous estimate of $v$ with those obtained by using the simpler ansatz (36). For $R_{\xi}$, if we only include the data with $L \geqslant 32$, we obtain $v=0.658(1)$. This suggests that a reliable estimate for $v$ might be $v=0.64(2)$. Using the same criterion for $J_{c}$, $R_{\xi}^{*}$, and $U^{*}$, we obtain the estimates reported in Table II. The scaling plot of $R_{\xi}$ is reported in Fig. 16. Scaling deviations are clearly visible for $L=16$. We have also determined the exponent $\eta_{q}$. The susceptibility has been fitted to the ansatz (38). The $\chi^{2}$ is not very sensitive to $\omega$ and is essentially
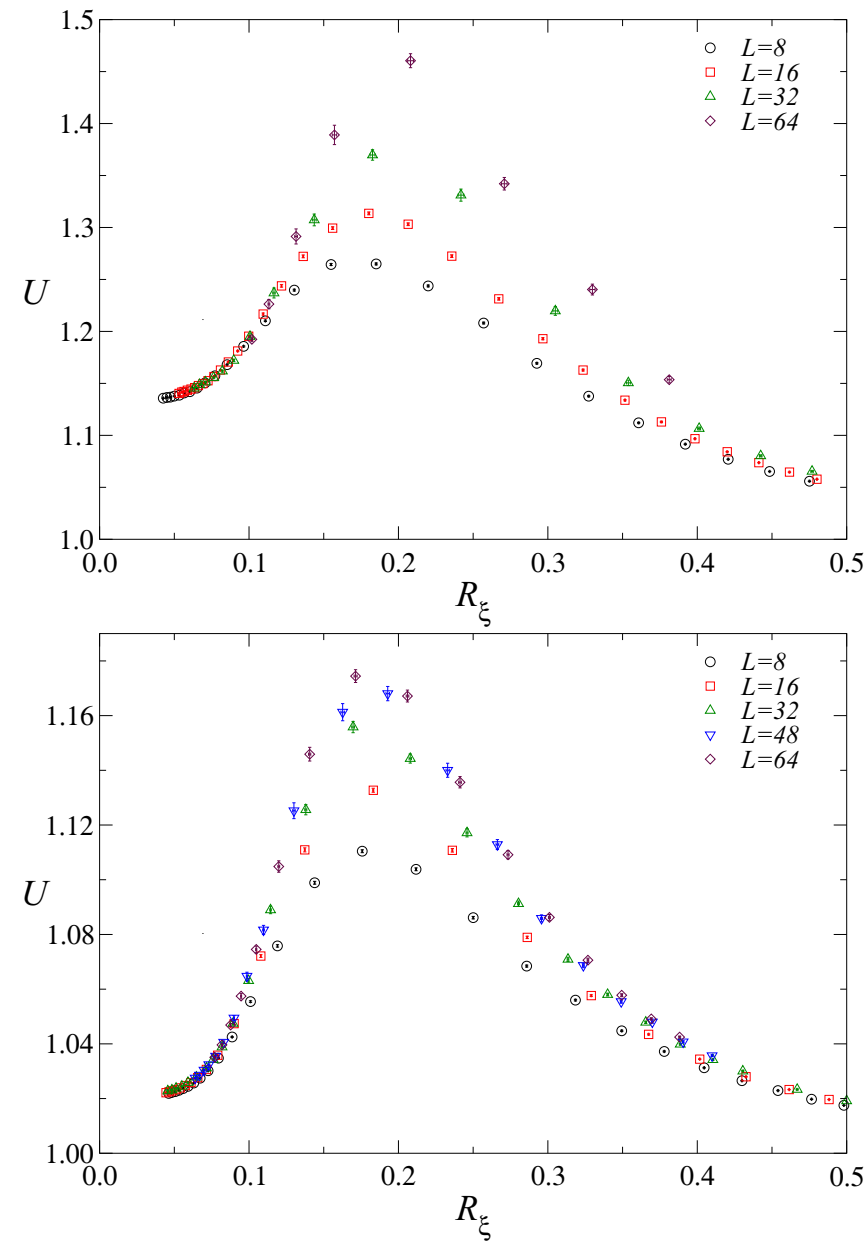

FIG. 15. Top: Estimates of $U$ versus $R_{\xi}$ for the $N=4$ lattice AH model at $\kappa=0.4$. Bottom: Estimates of $U$ versus $R_{\xi}$ for the $N=10$ lattice $\mathrm{AH}$ model at $\kappa=0.4$.

constant for $\omega \gtrsim 1.4$. Correspondingly, $\eta_{q}$ varies between $0.745(4)$ and $0.759(2)$. However, note that we expect $\omega \approx 1$ and thus we have conservatively considered the larger interval $\omega \gtrsim 0.8$. Since $\eta_{q}=0.727(6)$ for $\omega=0.8$, we end up with the final estimate $\eta_{q}=0.74(2)$. The quality of the fit is excellent (see Fig. 16).

In conclusion, the numerical results for $N=4$ and $N=10$ allow us to conclude that

$$
4<N_{\ell}<10 .
$$

A more precise determination of $N_{\ell}$ would require a substantially bigger computational effort, so that we do not pursue this issue further.

The upper bound on $N_{\ell}$ provides an upper bound on the number $N_{c}$, the smaller value of $N$ for which the 3D AH field theory has a stable fixed point, as discussed in Sec. III A. If we assume that the transitions observed for $N \geqslant 10 \mathrm{can}$ all be associated with the field theory stable fixed point-we provided evidence for that in the previous sections-we can conclude that

$$
N_{c}<10 \text {. }
$$



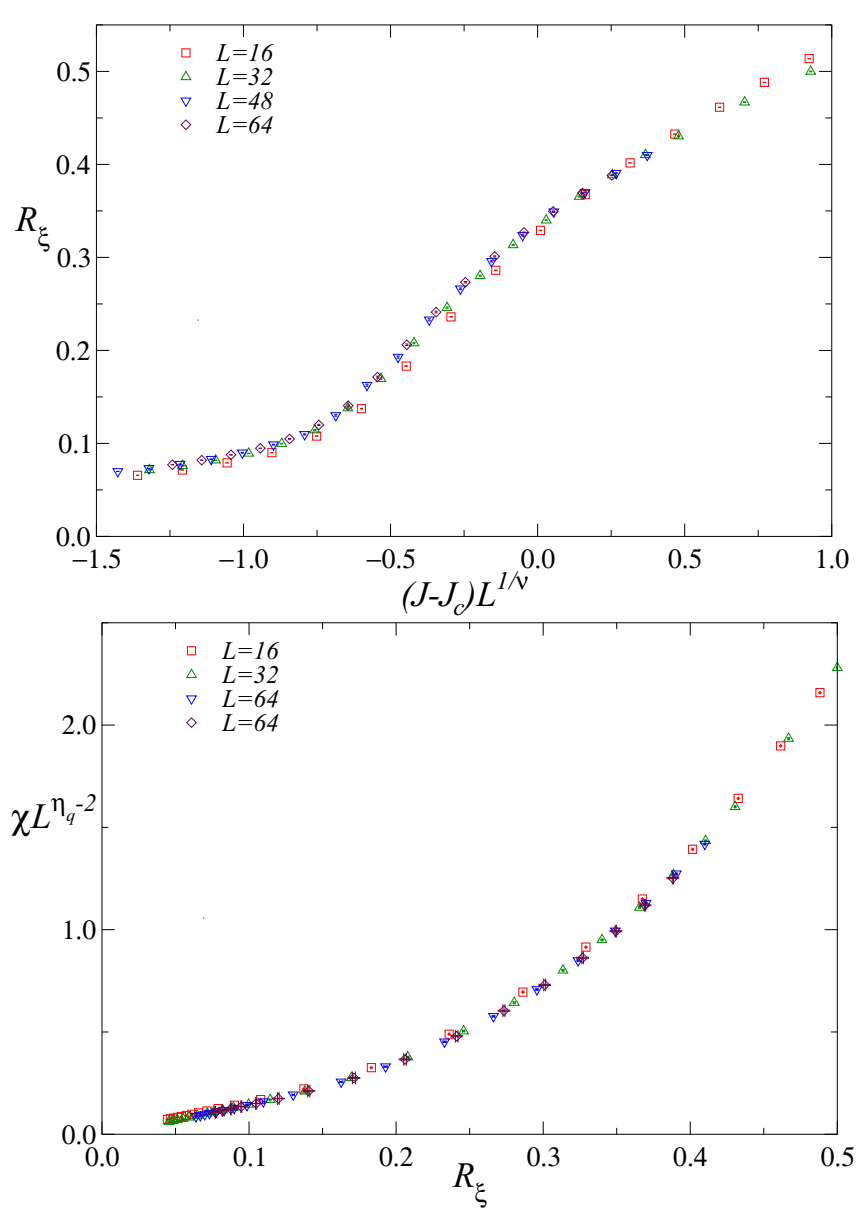

FIG. 16. Top: Plot of $R_{\xi}$ versus $\left(J-J_{c}\right) L^{1 / v}$; we use $J_{c}=$ 0.32187 and $v=0.64$. Bottom: Plot of $\chi L^{\eta_{q}-2}$ versus $R_{\xi}$; we use $\eta_{q}=0.74$. Results for the $N=10$ lattice AH model at $\kappa=0.4$, for several lattice sizes up to $L=64$.

On the other hand, the lower bound on $N_{\ell}$ cannot be straightforwardly extended to $N_{c}$. Indeed, the evidence of a first-order transition for a lattice model does not exclude the possibility that the corresponding field theory has a stable fixed point, since the given lattice model might be outside its attraction domain. We finally note that the bound Eq. (40) is in substantial agreement with the estimate $N_{c}=12.2(3.9)$ obtained by the analysis of the four-loop $\varepsilon$ expansion [43], mentioned in Sec. III A.

\section{CONCLUSIONS}

We have investigated the phase diagram and nature of the phase transitions of the $3 \mathrm{D}$ multicomponent lattice $\mathrm{AH}$ model with noncompact gauge fields. Our study confirms the existence of significant differences with the lattice AH model with compact gauge fields, both for small and large values of $N$ [38]. As sketched in Fig. 1, the phase diagram of the noncompact model with $N \geqslant 2$ is generally characterized by three phases: (i) the Coulomb phase, where the global $\mathrm{SU}(N)$ symmetry is unbroken and the electromagnetic correlations are long ranged; (ii) the Higgs phase, where the local bilinear operator $Q_{x}$ [cf. Eq. (8)] condenses, breaking the global $\mathrm{SU}(N)$ symmetry, and electromagnetic correlations are gapped; and (iii) a mixed molecular phase, where the local bilinear $Q_{x}$ condenses, but the electromagnetic field remains ungapped. We recall that the phase diagram of the compact lattice AH model presents only two phases. They are characterized by the condensation of the bilinear operator $Q_{x}$, while gauge fields are always in the confined phase [38].

We have studied in detail the Coulomb-to-Higgs transition line that ends at the $\mathrm{O}(2 N)$ transition point for $\kappa=\infty$ (i.e., for vanishing gauge coupling), and the Coulomb-to-molecular transition line that ends at the $\mathrm{CP}^{N-1}$ transition. Transitions along the $\mathrm{CH}$ line, if continuous, are expected to be associated with the stable fixed point of the RG flow of the continuum AH model (1). On the other hand, transitions along the CM line should be described by the LGW theory (20), because gauge correlations do not play a relevant role.

We summarize the behavior along the three transition lines as follows:

(i) The $\mathrm{CH}$ transitions separating the Coulomb and Higgs phases are weak first-order transitions for $N=2$. The same behavior is expected for sufficiently small $N$. Indeed, a firstorder transition is observed for $N=4$. As $N$ increases, the transitions become continuous, at least not too close to the multicritical point. We observe continuous transitions for $N \geqslant 10$. The corresponding critical behavior turns out to belong to the universality class of the stable fixed point of the continuum AH field theory, which predicts a continuous transition only for a large number of components, and in particular in the large- $N$ limit. Our numerical results provide a bound on the number $N_{c}$ defined in Sec. III A, which separates the small$N$ first-order transition regime from the large- $N$ continuous transition regime predicted by the 3D AH field theory. We obtain the upper bound $N_{c}<10$. If we further assume that the absence of continuous transitions along the $\mathrm{CH}$ transition line of the model considered in this paper corresponds to the absence of stable fixed points of the continuum AH field theory, we may speculate that $N_{c}$ belongs to the interval $4<N_{c}<10$.

(ii) For $N=2$ the $\mathrm{CM}$ transitions separating the Coulomb and molecular phase are continuous and belong to the $\mathrm{O}(3)$ vector universality class, as predicted by the LGW theory (20), for sufficiently small values of the inverse gauge coupling $\kappa$. As $\kappa$ increases along the CM line, the transition should eventually become of first order, as all transitions are expected to be of first order close to the point where the three transition lines meet. For $N \geqslant 3$, the $\mathrm{CM}$ transitions are expected to be of first order, as predicted by the LGW theory.

(iii) The transitions along the $\mathrm{MH}$ line are expected to be continuous, and to belong to the $\mathrm{XY}$ universality class, at least for sufficiently large values of the parameter $J$. However, we have not presented results along this transition line.

The identification of the large- $N$ continuous $\mathrm{CH}$ transitions with the universality class of the stable fixed point of the $\mathrm{AH}$ model is strongly supported by the excellent agreement of the numerical results for the critical exponent $v$ and $\eta$ with the predictions obtained using the $1 / N$ expansion within the continuum AH model. For instance, for $N=25$ we find $v=$ $0.802(8)$ and $\eta_{q}=0.883(7)$, to be compared with the large- $N$ estimates $v=0.805$ and $\eta_{q}=0.870$. As far as we know, this is the first quantitative evidence of such a correspondence.

It is worth comparing these results with those reported in Ref. [18] for a lattice $\mathrm{CP}^{N-1}$ model without monopoles. 
Numerical results for the monopole-free $\mathrm{CP}^{N-1}$ model provided clear evidence of a continuous transition for $N=25$ [59], at variance with what happens in the standard $\mathrm{CP}^{N-1}$, where the transition is discontinuous for any $N \geqslant 3$. Reference [18] conjectured that the transition for $N=25$ in the monopole-free model might be associated with the large- $N$ stable fixed point of the continuum AH field theory. The results of the present paper rule out this conjecture. The estimate of the critical exponent [18] $v=0.595(15)$ for the $N=$ 25 monopole-free model definitely disagrees with the result $v=0.802(8)$ obtained for the $N=25$ noncompact lattice $\mathrm{AH}$ model, which is instead in agreement with the large- $N$ expansion of the continuum AH field theory. Thus, we conclude that transitions of the large- $N$ monopole-free $\mathrm{CP}^{N-1}$ model are not described by the continuum AH field theory. It is tempting to conjecture that the reason for the difference is in the nature of the coexisting phases at the transition. The AH field theory is appropriate to describe transitions between a Coulomb and a Higgs phase, but it is not appropriate to describe the transition in the monopole-free $\mathrm{CP}^{N-1}$ model. Indeed, in the latter case no Higgs phase exists: A disordered monopole-free high-temperature phase coexists with a molecular phase in which electromagnetic modes are still ungapped.

\section{ACKNOWLEDGMENTS}

Numerical simulations have been performed on the CSN4 cluster of the Scientific Computing Center at INFN-PISA.

\section{APPENDIX: $C^{*}$ BOUNDARY CONDITIONS}

We wish now to discuss the role that boundary conditions play in noncompact formulations. We consider a finite system of size $L$ in all directions. As already discussed in Sec. II, the partition function defined in Eq. (7) is ill defined: $Z=\infty$ for any $L$, because of gauge invariance. A standard way out consists in considering only gauge-invariant observables and in introducing a gauge fixing that eliminates all zero modes. Let us indicate symbolically with $G\left[A_{x, \mu}\right]=0$ a maximal gauge fixing: If $\left\{A_{x, \mu}\right\}$ is a configuration that satisfies the gauge-fixing condition, there is no gauge transformation such the gauge-transformed configuration also satisfies the gaugefixing condition. Considering a gauge-invariant operator $B$, one can hope to obtain a well-defined average value by defining

$$
\langle B\rangle=\frac{\sum_{A z} B \delta(G) e^{-\beta H}}{\sum_{A z} \delta(G) e^{-\beta H}} .
$$

Unfortunately, in the case of periodic boundary conditions, also this expression is ill defined.

To clarify this issue, let us first consider the gauge Hamiltonian $H_{g}$, Eq. (6). It is invariant under the local gauge transformation

$$
A_{\boldsymbol{r}, \mu}^{[\alpha]}=A_{\boldsymbol{r}, \mu}+\alpha(\boldsymbol{r}+\hat{\mu})-\alpha(\boldsymbol{r}),
$$

where $\alpha(\boldsymbol{r})$ is an arbitrary function satisfying periodic boundary conditions. The Hamiltonian $H_{g}$, however, is also invariant under the shift

$$
A_{\boldsymbol{r}, \mu} \rightarrow A_{\boldsymbol{r}, \mu}+c_{\mu},
$$

where $c_{\mu}$ is a direction-dependent constant. To clarify the role played by the shift (A3), it is convenient to introduce the noncompact Polyakov loop along the direction $\mu$, defined by

$$
P_{\boldsymbol{r}_{\perp}, \mu}=\sum_{i=1}^{L} A_{\left(i, \boldsymbol{r}_{\perp}\right), \mu} .
$$

A generic point $\boldsymbol{r}$ is denoted with $\left(i, \boldsymbol{r}_{\perp}\right)$ where $r_{\mu}=i$, and $\boldsymbol{r}_{\perp}$ stands for the components of $\boldsymbol{r}$ different from the $\mu$ th one. It is immediate to verify that the noncompact Polyakov loop is gauge invariant, while

$$
P_{\boldsymbol{r}_{\perp}, \mu} \rightarrow P_{\boldsymbol{r}_{\perp}, \mu}+L c_{\mu}
$$

under the transformation (A3). This shows that the shift transformation cannot be rewritten as a gauge transformation. As a consequence there are three zero modes that cannot be eliminated by the introduction of a gauge fixing. Therefore, in the absence of the spin variables, also Eq. (A1) is ill defined.

The transformation (A3) is also present in compact formulations. It corresponds to $\lambda_{x, \mu} \rightarrow \lambda_{x, \mu} e^{i c_{\mu}}$. However, in this case the integration domain is compact and, therefore, zero modes do not make average values ill defined. This is obviously also the case of gauge transformations and, indeed, in the compact case no gauge fixing is needed to define rigorously the model.

The shift symmetry is broken when the spin fields $z_{\boldsymbol{x}, \mu}$ are added. However, because the gauge coupling of the spins is obtained through the fields $\lambda_{\boldsymbol{x}, \mu}$, transformations such that $e^{i c_{\mu}}=1$ leave the full Hamiltonian invariant. Therefore, the infinite discrete subgroup of transformations

$$
A_{\boldsymbol{r}, \mu} \rightarrow A_{\boldsymbol{r}, \mu}+2 \pi n_{\mu}, \quad n_{\mu} \in \mathbb{Z},
$$

is an invariance of the model, making expressions like Eq. (A1) ill defined. An identical problem is encountered in lattice quantum chromodynamics when studying the electromagnetic properties of hadrons using a noncompact formulation for the photon field (see, e.g., Refs. [87,88]).

To solve the problems mentioned above, we now discuss the $C^{*}$ boundary conditions proposed in Ref. [40]. For the system studied in this work, the $C^{*}$ boundary conditions are defined by the relations

$$
A_{r+L \hat{v}, \mu}=-A_{r, \mu}, \quad z_{r+L \hat{v}}=\bar{z}_{r} .
$$

For consistency with Eq. (A7), the function $\alpha(\boldsymbol{r})$ entering local gauge transformations,

$$
\begin{array}{rrr}
A_{\boldsymbol{r}, \mu}^{[\alpha]}= & A_{\boldsymbol{r}, \mu}+\alpha(\boldsymbol{r}+\hat{\mu})-\alpha(\boldsymbol{r}), \\
z_{\boldsymbol{r}}^{[\alpha]}= & \exp [-i \alpha(\boldsymbol{r})] z_{\boldsymbol{r}},
\end{array}
$$

has to satisfy antiperiodic boundary conditions

$$
\alpha(\boldsymbol{r}+L \hat{v})=-\alpha(\boldsymbol{r}) .
$$

Moreover, from the relation $z_{r+L \hat{v}}=\bar{z}_{r}$, it follows that the global $U(1)$ symmetry is explicitly broken down to its $\mathbb{Z}_{2}$ subgroup:

$$
A_{\boldsymbol{r}, \mu}^{[\alpha]}=A_{\boldsymbol{r}, \mu}, \quad z_{\boldsymbol{r}}^{[\alpha]}=s z_{\boldsymbol{r}},
$$

with $s= \pm 1$. Note that $C^{*}$ boundary conditions do not break translational invariance, but care should be taken when 
performing Fourier transforms. For instance, the field $Q^{a b}$ is periodic, while the plaquette operator is antiperiodic.

We will now show how to rewrite the previous conditions using only the fields that belong to the cubic lattice $[1, L]^{3}$, changing the form of the Hamiltonian for the sites and links close to the boundary. This is necessary for the MC implementation. Let us first consider the gauge transformations: The transformation law of the scalar fields in Eqs. (A8) does not require any modification, just like the transformation rule of the gauge field when $\boldsymbol{r}+\hat{\mu} \in[1, L]^{3}$. The gauge transformation of the fields $A_{r, \mu}$ on the boundary of the cube can instead be rewritten, using the antiperiodicity of $\alpha(\boldsymbol{r})$, in the form

$$
\begin{aligned}
& A_{1}^{[\alpha]}(L, a, b)=A_{1}(L, a, b)-\alpha(1, a, b)-\alpha(L, a, b), \\
& A_{2}^{[\alpha]}(a, L, b)=A_{2}(a, L, b)-\alpha(a, 1, b)-\alpha(a, L, b), \\
& A_{3}^{[\alpha]}(a, b, L)=A_{3}(a, b, L)-\alpha(a, b, 1)-\alpha(a, b, L),
\end{aligned}
$$

where $a, b \in[1, L]$.

The interaction term $H_{z}$ in Eq. (3) is written as a sum of terms which, for sites in the bulk of the lattice, are proportional to

$$
h_{\mu}(\boldsymbol{r})=\bar{z}(\boldsymbol{r}) \lambda_{\mu}(\boldsymbol{r}) \boldsymbol{z}(\boldsymbol{r}+\hat{\mu})+\text { c.c. }
$$

For sites on the boundary of the lattice instead, the interactions can be written as

$$
\begin{aligned}
& h_{1}(L, a, b)=\bar{z}(L, a, b) \lambda_{1}(L, a, b) \bar{z}(1, a, b)+\text { c.c., } \\
& h_{2}(a, L, b)=\bar{z}(a, L, b) \lambda_{2}(a, L, b) \bar{z}(a, 1, b)+\text { c.c., } \\
& h_{3}(a, b, L)=\bar{z}(a, b, L) \lambda_{3}(a, b, L) \bar{z}(a, b, 1)+\text { c.c. }
\end{aligned}
$$

These are the terms that explicitly break the global U(1) invariance. They are still gauge invariant, due to the different gauge transformations that are applied on the field $A_{r, \mu}$ on the boundary.

The noncompact gauge interaction term $H_{g}$ in Eq. (6) is written as a sum of terms involving the noncompact plaquette operator, which for sites in the bulk of the lattice can be written as

$$
\Pi_{\mu \nu}(\boldsymbol{r})=\left[A_{\mu}(\boldsymbol{r})+A_{\nu}(\boldsymbol{r}+\hat{\mu})-A_{\mu}(\boldsymbol{r}+\hat{v})-A_{\nu}(\boldsymbol{r})\right]^{2} .
$$

For plaquettes on the boundaries this expression has to be changed and we provide here explicit expressions for the case $(\mu, v)=(1,2)$ (the other two cases are completely analogous). For $a \in[1, L)$ and $b \in[1, L]$ we have to use

$$
\begin{aligned}
\Pi_{12}(L, a, b)= & {\left[A_{1}(L, a, b)-A_{2}(1, a, b)\right.} \\
& \left.-A_{1}(L, a+1, b)-A_{2}(L, a, b)\right]^{2}, \\
\Pi_{12}(a, L, b)= & {\left[A_{1}(a, L, b)+A_{2}(a+1, L, b)\right.} \\
& \left.+A_{1}(a, 1, b)-A_{2}(a, L, b)\right]^{2}, \\
\Pi_{12}(L, L, b)= & {\left[A_{1}(L, L, b)-A_{2}(1, L, b)\right.} \\
& \left.+A_{1}(L, 1, b)-A_{2}(L, L, b)\right]^{2},
\end{aligned}
$$

which are easily shown to be gauge invariant.

Let us now show that $C^{*}$ boundary conditions eliminate the shift symmetry that makes periodic boundary conditions ill defined. Indeed, in the $C^{*}$ case, Polyakov loops are not gauge invariant. Using for definiteness the Polyakov loop in the $\hat{z}$ direction, i.e.,

$$
P_{3}(x, y)=\sum_{z=1}^{L} A_{3}(x, y, z),
$$

we have

$$
P_{3}^{[\alpha]}(x, y)=P_{3}(x, y)-2 \alpha(x, y, 1) .
$$

A simple consequence of this fact is that, by means of local gauge transformations, we can enforce $A_{3}(\boldsymbol{r})=0$ for all points, obtaining the maximal temporal gauge (this is obviously not possible when using periodic boundary conditions, since Polyakov loops are gauge invariant in that case). The algorithm to implement the maximal temporal gauge is the following. We first perform a gauge transformation with $\alpha(x, y, 2)=-A_{3}(x, y, 1)$ and $\alpha(x, y, z)=0$ for $z \neq 2$ [this fixes $A_{3}(x, y, 1)=0$ ]; then we use a gauge transformation with $\alpha(x, y, 3)=-A_{3}(x, y, 2)$ and $\alpha(x, y, z)=0$ for $z \neq 3$ and so on, until we reach the plane $z=L$. At this point only $A_{3}(x, y, L)$ is not vanishing and we perform a transformation with $\alpha(x, y, z)=A_{3}(x, y, L) / 2$ (the same for all $z$ values).

To conclude the proof that $C^{*}$ boundary conditions make the gauge-fixed theory well defined, let us show that, once the maximal temporal gauge is introduced, there is a unique minimum of the gauge Hamiltonian $H_{g}$, confirming the absence of dangerous zero modes. Starting from

$$
\Pi_{13}(x, y, 1)=\left[A_{1}(x, y, 1)-A_{1}(x, y, 2)\right]^{2},
$$

by minimization we obtain $A_{1}(x, y, 2)=A_{1}(x, y, 1)$ for all $x, y$. If we now consider $\Pi_{13}(x, y, 2)$, we obtain in the same way $A_{1}(x, y, 3)=A_{1}(x, y, 2)$, and repeating the same procedure for $\Pi_{13}(x, y, z)$ with $1 \leqslant z \leqslant L-1$, we get $A_{1}(x, y, z)=$ $A_{1}(x, y, 1)$ for all $x, y, z$. The minimization of the boundary plaquette,

$$
\begin{aligned}
\Pi_{13}(x, y, L) & =\left[A_{1}(x, y, 1)+A_{1}(x, y, L)\right]^{2} \\
& =4 A_{1}(x, y, 1)^{2},
\end{aligned}
$$

finally implies $A_{1}(x, y, 1)=0$; hence $A_{1}(x, y, z)=0$ for all $x, y, z$. Using $\Pi_{23}$ instead of $\Pi_{13}$ the same argument shows that $A_{2}(x, y, z)=0$. We have therefore proved that, at variance with the case of periodic boundary conditions, when using $C^{*}$ boundary conditions, there is a single configuration (up to gauge transformations) that minimizes the gauge Hamiltonian: No gauge-invariant zero modes are present.

To conclude the Appendix, let us go back to the question of the gauge fixing. We have proved that the statistical averages are well defined provided that $C^{*}$ boundary conditions and a maximal gauge fixing are used. However, in the simulation we have not introduced any gauge fixing. We wish now to explain why the gauge fixing is irrelevant in MC calculations of gauge-invariant observables. Let us collectively call $\phi_{t}$ the fields we have generated at MC time $t$. There is obviously a gauge transformation that maps $\phi_{t}$ onto new fields $\phi_{t}^{[\alpha]}$ that satisfy the gauge-fixing condition: The correspondence between $\phi_{t}$ and $\phi_{t}^{[\alpha]}$ is unique. Therefore, the dynamics $\phi_{1} \rightarrow \phi_{2} \rightarrow \phi_{3} \cdots$ can be mapped onto the dynamics $\phi_{1}^{[\alpha]} \rightarrow \phi_{2}^{[\alpha]} \rightarrow \phi_{3}^{[\alpha]} \cdots$. Thus, even if we do not implement the gauge-fixing condition, gauge-invariant quantities take the same values as if they were obtained in 
a simulation in the gauge-fixed model. This is, of course, not true for non-gauge-invariant quantities. For instance, the fields
$A_{x, \mu}$ perform a random walk and their absolute values increase with time: Their averages are not defined.
[1] N. Read and S. Sachdev, Spin-Peierls, valence-bond solid, and Néel ground states of low-dimensional quantum antiferromagnets, Phys. Rev. B 42, 4568 (1990).

[2] S. Takashima, I. Ichinose, and T. Matsui, $\mathrm{CP}^{1}+\mathrm{U}(1)$ lattice gauge theory in three dimensions: Phase structure, spins, gauge bosons, and instantons, Phys. Rev. B 72, 075112 (2005).

[3] S. Takashima, I. Ichinose, and T. Matsui, Deconfinement of spinons on critical points: Multiflavor $\mathrm{CP}^{1}+\mathrm{U}(1)$ lattice gauge theory in three dimension, Phys. Rev. B 73, 075119 (2006).

[4] R. K. Kaul, Quantum phase transitions in bilayer $\mathrm{SU}(N)$ antiferromagnets, Phys. Rev. B 85, 180411(R) (2012).

[5] R. K. Kaul and A. W. Sandvik, Lattice Model for the SU(N) Néel to Valence-Bond Solid Quantum Phase Transition at Large $N$, Phys. Rev. Lett. 108, 137201 (2012).

[6] M. S. Block, R. G. Melko, and R. K. Kaul, Fate of $\mathrm{CP}^{N-1}$ Fixed Point with $q$ Monopoles, Phys. Rev. Lett. 111, 137202 (2013).

[7] A. Nahum, J. T. Chalker, P. Serna, M. Ortuño, and A. M. Somoza, Deconfined Quantum Criticality, Scaling Violations, and Classical Loop Models, Phys. Rev. X 5, 041048 (2015).

[8] C. Wang, A. Nahum, M. A. Metliski, C. Xu, and T. Senthil, Deconfined Quantum Critical Points: Symmetries and Dualities, Phys. Rev. X 7, 031051 (2017).

[9] A. W. Sandvik, Evidence for Deconfined Quantum Criticality in a Two-Dimensional Heisenberg Model with Four-Spin Interactions, Phys. Rev. Lett. 98, 227202 (2007).

[10] R. G. Melko and R. K. Kaul, Scaling in the Fan of an Unconventional Quantum Critical Point, Phys. Rev. Lett. 100, 017203 (2008).

[11] F.-J. Jiang, M. Nyfeler, S. Chandrasekharan, and U.-J. Wiese, From an antiferromagnet to a valence bond solid: Evidence for a first-order phase transition, J. Stat. Mech. (2008) P02009.

[12] A. W. Sandvik, Continuous Quantum Phase Transition between an Antiferromagnet and a Valence-Bond Solid in Two Dimensions: Evidence for Logarithmic Corrections to Scaling, Phys. Rev. Lett. 104, 177201 (2010).

[13] K. Harada, T. Suzuki, T. Okubo, H. Matsuo, J. Lou, H. Watanabe, S. Todo, and N. Kawashima, Possibility of deconfined criticality in $\mathrm{SU}(N)$ Heisenberg models at small $N$, Phys. Rev. B 88, 220408(R) (2013).

[14] K. Chen, Y. Huang, Y. Deng, A. B. Kuklov, N. V. Prokof'ev, and B. V. Svistunov, Deconfined Criticality Flow in the Heisenberg Model with Ring-Exchange Interactions, Phys. Rev. Lett. 110, 185701 (2013).

[15] S. Pujari, K. Damle, and F. Alet, Nèel-State to Valence-BondSolid Transition on the Honeycomb Lattice: Evidence for Deconfined Criticality, Phys. Rev. Lett. 111, 087203 (2013).

[16] H. Shao, W. Guo, and A. W. Sandvik, Quantum criticality with two length scales, Science 352, 213 (2016).

[17] T. Senthil, L. Balents, S. Sachdev, A. Vishwanath, and M. P. A. Fisher, Quantum Criticality beyond the Landau-GinzburgWilson Paradigm, Phys. Rev. B 70, 144407 (2004).

[18] A. Pelissetto and E. Vicari, Three-dimensional monopole-free $\mathrm{CP}^{N-1}$ models, Phys. Rev. E 101, 062136 (2020).
[19] O. I. Motrunich and A. Vishwanath, Emergent photons and transitions in the $\mathrm{O}(3)$ sigma model with hedgehog suppression, Phys. Rev. B 70, 075104 (2004).

[20] G. Murthy and S. Sachdev, Actions of hedgehogs instantons in the disordered phase of $2+1$ dimensional $\mathrm{CP}^{N-1}$ model, Nucl. Phys. B 344, 557 (1990).

[21] A. B. Kuklov, N. V. Prokof'ev, B. V. Svistunov, and M. Troyer, Deconfined criticality, runaway flow in the two-component scalar electrodynamics and weak first-order superfluid-solid transitions, Ann. Phys. 321, 1602 (2006).

[22] O. I. Motrunich and A. Vishwanath, Comparative study of Higgs transition in one-component and two-component lattice superconductor models, arXiv:0805.1494.

[23] A. B. Kuklov, M. Matsumoto, N. V. Prokof'ev, B. V. Svistunov, and M. Troyer, Deconfined Criticality: Generic First-Order Transition in the SU(2) Symmetry Case, Phys. Rev. Lett. 101, 050405 (2008).

[24] A. B. Kuklov, M. Matsumoto, N. V. Prokof'ev, B. V. Svistunov, and M. Troyer, Comment on "Comparative study of Higgs transition in one-component and two-component lattice superconductor models, arXiv:0805.2578.

[25] D. Charrier, F. Alet, and P. Pujol, Gauge Theory Picture of an Ordering Transition in a Dimer Model, Phys. Rev. Lett. 101, 167205 (2008).

[26] J. Lou, A. W. Sandvik, and N. Kawashima, Antiferromagnetic to valence-bond-solid transitions in two-dimensional $\mathrm{SU}(\mathrm{N})$ Heisenberg models with multispin interactions, Phys. Rev. B 80, 180414(R) (2009).

[27] G. Chen, J. Gukelberger, S. Trebst, F. Alet, and L. Balents, Coulomb gas transitions in three-dimensional classical dimer models, Phys. Rev. B 80, 045112 (2009).

[28] D. Charrier and F. Alet, Phase diagram of an extended classical dimer model, Phys. Rev. B 82, 014429 (2010).

[29] A. Banerjee, K. Damle, and F. Alet, Impurity spin texture at a deconfined quantum critical point, Phys. Rev. B 82, 155139 (2010).

[30] E. V. Herland, T. A. Bojesen, E. Babaev, and A. Sudbø, Phase structure and phase transitions in a three-dimensional SU(2) superconductor, Phys. Rev. B 87, 134503 (2013).

[31] L. Bartosch, Corrections to scaling in the critical theory of deconfined criticality, Phys. Rev. B 88, 195140 (2013).

[32] T. A. Bojesen and A. Sudb $\varnothing$, Berry phases, current lattices, and suppression of phase transitions in a lattice gauge theory of quantum antiferromagnets, Phys. Rev. B 88, 094412 (2013).

[33] A. Nahum, P. Serna, J. T. Chalker, M. Ortuño, and A. M. Somoza, Emergent SO(5) Symmetry at the Néel to ValenceBond-Solid Transition, Phys. Rev. Lett. 115, 267203 (2015).

[34] G. J. Sreejith and S. Powell, Scaling dimensions of highercharge monopoles at deconfined critical points, Phys. Rev. B 92, 184413 (2015).

[35] P. Serna and A. Nahum, Emergence and spontaneous breaking of approximate $\mathrm{O}(4)$ symmetry at a weakly first-order deconfined phase transition, Phys. Rev. B 99, 195110 (2019). 
[36] A. W. Sandvik and B. Zhao, Consistent scaling exponents at the deconfined quantum-critical point, Chin. Phys. Lett. 37, 057502 (2020).

[37] F. D. M. Haldane, O(3) Nonlinear $\sigma$-Model and the Topological Distinction between Integer- and Half-Integer-Spin Antiferromagnets in Two Dimensions, Phys. Rev. Lett. 61, 1029 (1988).

[38] A. Pelissetto and E. Vicari, Multicomponent compact AbelianHiggs lattice models, Phys. Rev. E 100, 042134 (2019).

[39] A. S. Kronfeld and U. J. Wiese, SU(N) gauge theories with C periodic boundary conditions. 1. Topological structure, Nucl. Phys. B 357, 521 (1991).

[40] B. Lucini, A. Patella, A. Ramos and N. Tantalo, Charged hadrons in local finite-volume QED + QCD with $\mathrm{C}^{*}$ boundary conditions, J. High Energy Phys. 02 (2016) 076.

[41] B. I. Halperin, T. C. Lubensky, and S. K. Ma, First-Order Phase Transitions in Superconductors and Smectic-A Liquid Crystals, Phys. Rev. Lett. 32, 292 (1974).

[42] R. Folk and Y. Holovatch, On the critical fluctuations in superconductors, J. Phys. A 29, 3409 (1996).

[43] B. Ihrig, N. Zerf, P. Marquard, I. F. Herbut, and M. M. Scherer, Abelian Higgs model at four loops, fixed-point collision and deconfined criticality, Phys. Rev. B 100, 134507 (2019).

[44] G. Fejos and T. Hatsuda, Renormalization group flows of the $N$-component Abelian Higgs model, Phys. Rev. D 96, 056018 (2017).

[45] P. Di Vecchia, A. Holtkamp, R. Musto, F. Nicodemi, and R. Pettorino, Lattice $\mathrm{CP}^{N-1}$ models and their large- $N$ behaviour, Nucl. Phys. B 190, 719 (1981).

[46] V. Yu. Irkhin, A. A. Katanin, and M. I. Katsnelson, $1 / N$ expansion for critical exponents of magnetic phase transitions in the $\mathrm{CP}^{N-1}$ model for $2<d<4$, Phys. Rev. B 54, 11953 (1996).

[47] M. Moshe and J. Zinn-Justin, Quantum field theory in the large $N$ limit: A review, Phys. Rep. 385, 69 (2003).

[48] R. K. Kaul and S. Sachdev, Quantum criticality of U(1) gauge theories with fermionic and bosonic matter in two spatial dimensions, Phys. Rev. B 77, 155105 (2008).

[49] A. Pelissetto and E. Vicari, Critical phenomena and renormalization group theory, Phys. Rep. 368, 549 (2002).

[50] A. Pelissetto and E. Vicari, Three-dimensional ferromagnetic $\mathrm{CP}^{N-1}$ models, Phys. Rev. E 100, 022122 (2019).

[51] K. G. Wilson and J. Kogut, The renormalization group and the $\epsilon$ expansion, Phys. Rep. 12, 77 (1974).

[52] M. E. Fisher, The renormalization group in the theory of critical behavior, Rev. Mod. Phys. 47, 543 (1975).

[53] A. Pelissetto, A. Tripodo, and E. Vicari, Landau-GinzburgWilson approach to critical phenomena in the presence of gauge symmetries, Phys. Rev. D 96, 034505 (2017).

[54] C. Bonati, A. Pelissetto, and E. Vicari, Phase Diagram, Symmetry Breaking, and Critical Behavior of Three-Dimensional Lattice Multiflavor Scalar Chromodynamics, Phys. Rev. Lett. 123, 232002 (2019); C. Bonati, Three-dimensional lattice multiflavor scalar chromodynamics: Interplay between global and gauge symmetries, Phys. Rev. D 101, 034505 (2020).

[55] C. Bonati, A. Pelissetto, and E. Vicari, Three-dimensional phase transitions in multiflavor scalar $\mathrm{SO}\left(N_{c}\right)$ gauge theories, Phys. Rev. E 101, 062105 (2020).

[56] F. Delfino, A. Pelissetto, and E. Vicari, Three-dimensional antiferromagnetic $\mathrm{CP}^{N-1}$ models, Phys. Rev. E 91, 052109 (2015).
[57] A. Nahum, J. T. Chalker, P. Serna, M. Ortuño, and A. M. Somoza, 3D Loop Models and the $\mathrm{CP}^{N-1}$ Sigma Model, Phys. Rev. Lett. 107, 110601 (2011).

[58] A. Nahum, J. T. Chalker, P. Serna, M. Ortuño, and A. M. Somoza, Phase transitions in three-dimensional loop models and the $\mathrm{CP}^{N-1} \sigma$ model, Phys. Rev. B 88, 134411 (2013).

[59] A. Pelissetto and E. Vicari, Large- $N$ behavior of threedimensional lattice $\mathrm{CP}^{N-1}$ models, J. Stat. Mech.: Theory Exp. (2020) 033209.

[60] K. Kataoka, S. Hattori, and I. Ichinose, Effective field theory for $\operatorname{Sp}(N)$ antiferromagnets and their phase structure, Phys. Rev. B 83, 174449 (2011).

[61] M. Campostrini, A. Pelissetto, P. Rossi, and E. Vicari, Fourpoint renormalized coupling in $\mathrm{O}(N)$ models, Nucl. Phys. B 459, 207 (1996).

[62] H. G. Ballesteros, L. A. Fernandez, V. Martin-Mayor, and A. Munoz Sudupe, Finite size effects on measures of critical exponents in $d=3 \mathrm{O}(N)$ models, Phys. Lett. B 387, 125 (1996).

[63] P. Butera and M. Comi, $N$-vector spin models on the sc and the bcc lattices: A study of the critical behavior of the susceptibility and of the correlation length by high temperature series extended to order $\beta^{21}$, Phys. Rev. B 56, 8212 (1997).

[64] M. Hasenbusch, Monte Carlo study of a generalized icosahedral model on the simple cubic lattice, Phys. Rev. B 102, 024406 (2020).

[65] M. V. Kompaniets and E. Panzer, Minimally subtracted six-loop renormalization of $\phi^{4}$-symmetric theory and critical exponents, Phys. Rev. D 96, 036016 (2017).

[66] M. Hasenbusch and E. Vicari, Anisotropic perturbations in 3D O(N) vector models, Phys. Rev. B 84, 125136 (2011).

[67] M. Campostrini, M. Hasenbusch, A. Pelissetto, P. Rossi, and E. Vicari, Critical exponents and equation of state of the threedimensional Heisenberg universality class, Phys. Rev. B 65, 144520 (2002).

[68] R. Guida and J. Zinn-Justin, Critical exponents of the $N$-vector model, J. Phys. A 31, 8103 (1998).

[69] C. Dasgupta and B. I. Halperin, Phase Transitions in a Lattice Model of Superconductivity, Phys. Rev. Lett. 47, 1556 (1981).

[70] T. Neuhaus, A. Rajantie and K. Rummukainen, Numerical study of duality and universality in a frozen superconductor, Phys. Rev. B 67, 014525 (2003).

[71] D. Weston and E. Babaev, Vortices and composite order in $\mathrm{SU}(N)$ theories coupled to Abelian gauge field, arXiv:1908.10847.

[72] K.-S. Liu and M. E. Fisher, Quantum lattice gas and the existence of a supersolid, J. Low Temp. Phys. 10, 655 (1972).

[73] M. E. Fisher and D. R. Nelson, Spin Flop, Supersolids, and Bicritical and Tetracritical Points, Phys. Rev. Lett. 32, 1350 (1974).

[74] D. R. Nelson, J. M. Kosterlitz, and M. E. Fisher, Renormalization-Group Analysis of Bicritical and Tetracritical Points, Phys. Rev. Lett. 33, 813 (1974); J. M. Kosterlitz, D. R. Nelson, and M. E. Fisher, Bicritical and tetracritical points in anisotropic antiferromagnetic systems, Phys. Rev. B 13, 412 (1976). 
[75] P. Calabrese, A. Pelissetto, and E. Vicari, Multicritical behavior of $O\left(n_{1}\right) \oplus O\left(n_{2}\right)$-symmetric systems, Phys. Rev. B 67, 054505 (2003).

[76] M. Hasenbusch, A. Pelissetto, and E. Vicari, Instability of the $\mathrm{O}(5)$ critical behavior in the $\mathrm{SO}(5)$ theory of high- $T_{c}$ superconductors, Phys. Rev. B 72, 014532 (2005).

[77] M. S. S. Challa, D. P. Landau, and K. Binder, Finite-size effects at temperature-driven first-order transitions, Phys. Rev. B 34, 1841 (1986).

[78] K. Vollmayr, J. D. Reger, M. Scheucher, and K. Binder, Finite size effects at thermally-driven first order phase transitions: A phenomenological theory of the order parameter distribution, Z. Phys. B 91, 113 (1993).

[79] J. Lee and J. M. Kosterlitz, Finite-size scaling and Monte Carlo simulations of first-order phase transitions, Phys. Rev. B 43, 3265 (1991).

[80] P. Calabrese, P. Parruccini, A. Pelissetto, and E. Vicari, Critical behavior of $\mathrm{O}(2) \otimes \mathrm{O}(N)$-symmetric models, Phys. Rev. B 70, 174439 (2004).

[81] M. Campostrini, J. Nespolo, A. Pelissetto, and E. Vicari, FiniteSize Scaling at First-Order Quantum Transitions, Phys. Rev. Lett. 113, 070402 (2014).
[82] B. Nienhuis and M. Nauenberg, First-Order Phase Transitions in Renormalization-Group Theory, Phys. Rev. Lett. 35, 477 (1975).

[83] M. E. Fisher and A. N. Berker, Scaling for first-order phase transitions in thermodynamic and finite systems, Phys. Rev. B 26, 2507 (1982).

[84] V. Privman and M. E. Fisher, Finite-size effects at first-order transitions, J. Stat. Phys. 33, 385 (1983).

[85] H. Panagopoulos, A. Pelissetto, and E. Vicari, Dynamic scaling behavior at thermal first-order transitions in systems with disordered boundary conditions, Phys. Rev. D 98, 074507 (2018).

[86] A. Pelissetto, D. Rossini, and E. Vicari, Finite-size scaling at first-order quantum transitions when boundary conditions favor one of the two phases, Phys. Rev. E 98, 032124 (2018).

[87] M. Hayakawa and S. Uno, QED in finite volume and finite size scaling effect on electromagnetic properties of hadrons, Prog. Theor. Phys. 120, 413 (2008).

[88] S. Borsanyi, S. Durr, Z. Fodor, C. Hoelbling, S. D. Katz, S. Krieg, L. Lellouch, T. Lippert, A. Portelli, K. K. Szabo, and B. C. Toth, Ab initio calculation of the neutron-proton mass difference, Science 347, 1452 (2015). 